\title{
OBJECTIVE FUNCTION DESIGN FOR ROBUST OPTIMALITY OF LINEAR CONTROL UNDER STATE-CONSTRAINTS AND UNCERTAINTY
}

\author{
FABIO BAGAGIOLO ${ }^{1}$ AND DARIO BAUSO ${ }^{2}$
}

\begin{abstract}
We consider a model for the control of a linear network flow system with unknown but bounded demand and polytopic bounds on controlled flows. We are interested in the problem of finding a suitable objective function that makes robust optimal the policy represented by the so-called linear saturated feedback control. We regard the problem as a suitable differential game with switching cost and study it in the framework of the viscosity solutions theory for Bellman and Isaacs equations.
\end{abstract}

Mathematics Subject Classification. 49L25, 49N90, 90C35.

Received March 12, 2009. Revised July 27, 2009.

Published online October 30, 2009.

\section{INTRODUCTION}

In the recent work [3], the authors study the following problem: find an objective function $(\zeta, \mu, \omega) \mapsto$ $g(\zeta, \mu, \omega)$ such that the feedback linear saturated control $\bar{u}(t)=\operatorname{sat}(-k z(t))=\left(\bar{u}_{1}(t), \ldots, \bar{u}_{n}(t)\right)$ with $k>0$ and

$$
\bar{u}_{i}(t)=\operatorname{sat}\left(-k z_{i}(t)\right)= \begin{cases}1 & \text { if } z_{i}(t) \leq-\frac{1}{k} \\ -k z_{i}(t) & \text { if }\left|z_{i}(t)\right| \leq \frac{1}{k} \\ -1 & \text { if } z_{i}(t) \geq \frac{1}{k}\end{cases}
$$

is robustly optimal for the problem of minimizing

$$
J(\zeta, u, w)=\int_{0}^{+\infty} \mathrm{e}^{-t} g(z(t), u(t), w(t)) \mathrm{d} t
$$

subject to

$$
\left\{\begin{array}{l}
\dot{z}(t)=u(t)-D w(t), \quad t>0 \\
z(0)=\zeta
\end{array}\right.
$$

under the unknown disturbance $w(\cdot)$. Here, $z(\cdot) \in \mathbb{R}^{n}$ is the state variable, $\zeta \in \mathbb{R}^{n}$ is the initial state, $t \mapsto u(t)$ is the measurable control, taking value, for all $t \geq 0$, in the set of constant controls

$$
\mathcal{U}=\left\{\mu \in \mathbb{R}^{n}|| \mu_{i} \mid \leq 1 \forall i=1, \ldots, n\right\}
$$

\footnotetext{
Keywords and phrases. Optimal control, viscosity solutions, differential games, switching, flow control, networks.

${ }^{1}$ Dipartimento di Matematica, Università di Trento, Via Sommarive 14, 38050 Povo-Trento, Italy. bagagiol@science. unitn.it

2 DINFO, Università di Palermo, 90128 Palermo, Italy. dario.bauso@unipa.it
} 
$t \mapsto w(t)$ is the measurable unknown disturbance, taking value, for all $t \geq 0$, in the set of constant disturbances

$$
\mathcal{W}=\left\{\omega \in \mathbb{R}^{m}|| \omega_{j} \mid \leq 1 \forall j=1, \ldots, m\right\},
$$

and finally $D$ is a given constant $n \times m$ matrix.

Such a problem arises from a model of linear network flow system with unknown but bounded demand and polytopic bounds on controlled flows (see Bauso et al. [6] and the present Sect. 1). In particular, the following compact embedding hypothesis

$$
D \mathcal{W}=\{D \omega \mid \omega \in \mathcal{W}\} \subset \subset \mathcal{U},
$$

guarantees that, if the linear saturated control $\bar{u}$ is used, the state $z(\cdot)$ reaches the target

$$
\mathcal{T}=\left\{\zeta \in \mathbb{R}^{n}|| \zeta_{i} \mid \leq \frac{1}{k} \forall i=1, \ldots, n\right\}
$$

in finite time and, once $\mathcal{T}$ is reached, it will remain inside $\mathcal{T}$ for all the times. And this happens whichever the disturbance $w(\cdot)$ is.

The problem of driving the state $z(\cdot)$ into the target $\mathcal{T}$ is called $\epsilon$-stabilizability problem of $z(\cdot)$, where $\epsilon$ is the maximal size of $\mathcal{T}$.

For the modeling meaning of the target set $\mathcal{T}$ see again [6] and Section 1.

In [3] the problem is firstly addressed giving a suitable meaning to the robust optimality of the linear saturated control. This is done by a "differential game" approach in the sense of lower value of the game (see Elliot and Kalton [16]). We interpret the problem as a game between a first player who wants to minimize the payoff and uses the control $u$, and a second player who wants to maximize the payoff and uses the control $w$. Let us define the following sets for the measurable controls $u$ and $w$

$$
\begin{aligned}
& U=\{u:[0,+\infty[\rightarrow \mathcal{U} \mid u \text { measurable }\}, \\
& W=\{w:[0,+\infty[\rightarrow \mathcal{W} \mid w \text { measurable }\},
\end{aligned}
$$

and the set of the nonanticipating strategies for the first player

$$
\begin{aligned}
& \Gamma=\{\gamma=\gamma[\cdot]: W \rightarrow U \mid \\
& w_{1}(s)=w_{2}(s) \forall s \in[0, t] \Longrightarrow \gamma\left[w_{1}\right](s)=\gamma\left[w_{2}\right](s) \forall s \in[0, t], \\
& \left.\forall w_{1}, w_{2} \in W, \forall t \geq 0\right\} .
\end{aligned}
$$

For every initial state $\zeta$ fixed, we regard the linear saturated control $\bar{u}$ as a particular nonanticipating strategy $\gamma_{\zeta}$ for the first player

$$
\forall w \in W, \forall t \in\left[0,+\infty\left[, \quad \gamma_{\zeta}[w](t)=\operatorname{sat}(-k z(t)),\right.\right.
$$

where $z(t)$ is exactly the solution of (0.2) at the time $t$ with $w$ and $\bar{u}=$ sat as controls, i.e.

$$
\left\{\begin{array}{l}
\dot{z}(t)=\operatorname{sat}(-k z(t))-D w(t), \\
z(0)=\zeta .
\end{array}\right.
$$

Hence, we consider the following two problems (respectively maximization problem and minmax problem)

$$
\begin{aligned}
& \sup _{w \in W} J\left(\zeta, \gamma_{\zeta}[w], w\right)=V(\zeta), \\
& \text { subject to } \dot{z}(t)=\gamma_{\zeta}[w](t)-D w(t), z(0)=\zeta,
\end{aligned}
$$


and

where the payoff $J$ is given in $(0.1)$.

$$
\begin{aligned}
& \inf _{\gamma \in \Gamma} \sup _{w \in W} J(\zeta, \gamma[w], w)=\tilde{V}(\zeta), \\
& \text { subject to } \dot{z}(t)=\gamma[w](t)-D w(t), z(0)=\zeta,
\end{aligned}
$$

Definition 0.1. We say that the linear saturated control is robustly optimal if $V(\zeta)=\tilde{V}(\zeta)$ for all $\zeta \in \mathbb{R}^{n}$, that is if the value function of the maximization problem in $w$ with fixed strategy $\gamma_{\zeta}$ is equal to the lower value function (see Elliot and Kalton [16]) of the differential game given by minimization in the control $u$ and maximization in the unknown disturbance $w$.

The way to prove the equality between $V$ and $\tilde{V}$ used in [3] is to consider respectively the Hamilton-JacobiBellman and the Hamilton-Jacobi-Isaacs equations that they must respectively solve in the viscosity sense (see Crandall et al. [14] and Bardi and Capuzzo Dolcetta [4]). Such equations are respectively, neglecting for the moment possible boundary conditions,

$$
\begin{aligned}
& V(\zeta)+H(\zeta, \nabla V(\zeta))=0 \\
& \tilde{V}(\zeta)+\tilde{H}(\zeta, \nabla \tilde{V}(\zeta))=0,
\end{aligned}
$$

where $\nabla$ is the gradient, and the Hamiltonians $H$ and $\tilde{H}$ are defined, for all $\zeta \in \mathbb{R}^{n}, p \in \mathbb{R}^{n}$, as

$$
\begin{aligned}
& H(\zeta, p)=\min _{\omega \in \mathcal{W}}\{-(\operatorname{sat}(-k \zeta)-D \omega) \cdot p-g(\zeta, \operatorname{sat}(-k \zeta), \omega)\}, \\
& \tilde{H}(\zeta, p)=\min _{\omega \in \mathcal{W}} \max _{\mu \in \mathcal{U}}\{-(\mu-D \omega) \cdot p-g(\zeta, \mu, \omega)\} .
\end{aligned}
$$

In [3] we actually exhibit a function $\phi$ solving both (0.11)-(0.12), and, using uniqueness results for (0.11)-(0.12) we conclude $\phi=V=\tilde{V}$. However, apart from a simpler one-dimensional case, it seems suitable to split the problem into two different problems: one outside the target $\mathcal{T}$, giving it a "minimum-time" feature, and one inside the target, giving it a linear-quadratic feature. For the second case, some restriction on the behavior of the time-dependent disturbance $w(\cdot)$ is forced by choosing some suitable forcing terms in $g$.

In the present work instead, we address the problem inside the target only, for which the saturated control is then linear and which presents state-constraints. Moreover we relax some of the forcing terms considered in [3]. To this end, as explained next, it seems necessary to introduce a discontinuity (switching term) in the objective function $g$, and this fact may lead to some problem for the uniqueness of the Hamilton-Jacobi equations. To overcome such a difficulty, we approximate that switching term by a delayed thermostat and then study a suitable kind of hybrid system, in the spirit of Bagagiolo [1] (see Fig. 7). This leads to the introduction of two new state-variables: the switching one and a variable which counts switchings. Moreover, in order to get uniqueness for both Hamilton-Jacobi problems, these are casted into boundary value problems, which correspond to an optimal control problem and to a differential game with exit-cost. In particular, the exit-time differential game problem is not well present in the literature. Hence, some suitable new results for such a problem are also here reported. For another dynamic programming approach to some particular kinds of hybrid differential games, see for instance Dharmatti and Ramaswamy [15].

The problem here described, may be viewed as an inverse problem in optimal control: find and objective function such that a given control turns out to be optimal. Up to our knowledge, there are only few works on such a subject, see for instance Casti [12], Lee and Liaw [22], Larin [21]. Except for [12], all of them are concerning linear systems with unbounded state-space and unbounded controls. They look for a quadratic objective function by means of searching for suitable matrices solving a Riccati-type equation. Our problem is concerning a linear system too, but we also consider state-constraints and moreover we have restriction on the control set. In [12] a nonlinear problem is instead considered. Under hypotheses of smoothness, using dynamic programming, a partial differential equation satisfied by the objective function is guessed. However, also in this case, there are no constraints in the state and in the control, and no uniqueness results for the Hamilton-Jacobi equation are used. 
Note that our study also addresses the problem of differential games. Moreover, we also look for a "good shaped" objective function $g$ such that the value $V=\tilde{V}$ is good shaped too (for instance prevalently quadratic).

The paper is organized as follows. In Section 1 we briefly outline the applicative motivations for the model (0.2) and for our searching the objective function $g$ which makes sat robust. In Section 2 we outline the reasoning that leads to guess a suitable objective function. In Section 3 we rigorous formulate both the maximization and the minmax problem, and give the suitable uniqueness results. In Section 4 we deduce the robustness of the linear saturated control. In Section 5 we illustrate some numerical simulations. In the Appendix 6 we give some basic facts and some new suitable results about optimal control/differential game problems, dynamic programming, Hamilton-Jacobi equations and viscosity solutions. Moreover, we also outline some basic facts on the analytical representation of the delayed thermostatic switching rule.

\section{Motivations}

The idea of finding an objective function such that the feedback linear saturated control is robustly optimal is in the spirit of mechanism design, or inverse game theory. Indeed, the main topic of the mechanism design is the definition of game rules or incentive schemes that induce self-interested players to cooperate and reach Pareto optimal solutions.

The system used in this work is similar in spirit with [6] and most references therein where saturated control and unknown but bounded demands are also addressed. There, the authors derive for the first time the linear system (0.2) starting from a standard network flow system (see dynamics (1.1) below) with bounding sets $(0.3)-(0.4)$ and formulate the $\epsilon$-stabilizability problem for $z(t)$ as an auxiliary problem to solve a network flow control problem under input average constraints as recalled carefully next. The saturated control policy is proved to solve the $\epsilon$-stabilizability problem in [6].

Our interest for the saturated control is due to the fact that (i) it solves the $\epsilon$-stabilizability problem in [6]; (ii) it represents the simplest form of a piece-wise linear control the latter playing a central role in multiparametric optimization [7].

As regards the perturbation description, the idea of modeling the demand as unknown but bounded variable is in line with some recent literature on robust optimization $[8,10,13,20]$ though the "unknown but bounded" approach has a long history in control [9]. In particular, in [20] the authors deal with a problem of the same nature of the one addressed here (they call it terminal linear quadratic control problem), with the only difference that they do not take into account input constraints.

We also wish to highlight the analogies between the notion of feedback in control, present in this work, and the notion of recourse used in robust optimization (see e.g., [13]) where some variables are function of the perturbation realization. We also find that in [13], the linear saturated control is dealt with under the different name of deflected linear decision rule.

\subsection{Why model (0.2)? Linear network flow systems}

Let $G=(V, E)$ a graph with $|V|=n$ nodes and $|E|=m$ arcs. The graph $G$ describes the topology of a flow network system. The system manager controls the flows (of materials) of the $m$ arcs in order to meet the demand materializing at the $n$ nodes. Nodes model the different sites where inventory is stored. If we denote by $B \in \mathbb{R}^{n \times m}$ the incidence matrix of the graph, dynamics of the inventory $x(t)$ at the nodes is

$$
\dot{x}(t)=B u(t)-w(t) .
$$

The state $x(t)$ integrates the deviation between the demand and the flow arriving to and departing from the nodes. Previous studies (see [6]) show the connection between the $\epsilon$-stabilizability problem of $z(t)$ and the $\epsilon$-stabilizability problem of $x(t)$ with the additional requirement of satisfying certain average constraints on $u(t)$, 
discussed below. To highlight such a relation, take a $D \in \mathbb{R}^{n \times m}$ satisfying

$$
\begin{array}{r}
B D=I \\
\mathcal{U} \supset D \mathcal{W} .
\end{array}
$$

We can interpret matrix $D$ as follows: the $i$ th row of $D$ describes how to partition the demand at the $i$ th node among the arcs entering or leaving the nodes. Now, complete matrices $B$ and $D$ with matrices $C$ and $F$ such that

$$
\left[\begin{array}{l}
B \\
C
\end{array}\right]\left[\begin{array}{ll}
D & F
\end{array}\right]=I
$$

Consider the augmented system

$$
\begin{aligned}
& \dot{x}(t)=B u(t)-w(t) \\
& \dot{y}(t)=C u(t),
\end{aligned}
$$

and consider the new variable $z(t)$ defined as

$$
z(t)=\left[\begin{array}{ll}
D & F
\end{array}\right]\left[\begin{array}{l}
x(t) \\
y(t)
\end{array}\right], \quad\left[\begin{array}{l}
x(t) \\
y(t)
\end{array}\right]=\left[\begin{array}{l}
B \\
C
\end{array}\right] z(t) .
$$

By differentiating $z$ we obtain $\dot{z}(t)=u(t)-D w(t)$, which is exactly dynamics (0.2). Now, with $z$ defined as in (1.6), any control that $\epsilon$-stabilizes $z(t) \epsilon$-stabilizes $x(t)$ as well and also implies

$$
\lim _{T \rightarrow \infty} A v[w]=0 \Rightarrow \lim _{T \rightarrow \infty} A v[u]=0
$$

where $A v[w]:=\frac{1}{T} \int_{0}^{T} w(t) \mathrm{d} t$ and $A v[u]:=\frac{1}{T} \int_{0}^{T} u(t) \mathrm{d} t$. The above condition, known as average constraint, means that if the long-term average of the demand is null then also the long-term average of the control is null. To see this, observe that by integrating the left and right term of $(0.2)$ we have that

$$
\lim _{T \rightarrow \infty} \frac{z(T)-z(0)}{T}=\lim _{T \rightarrow \infty} \frac{1}{T} \int_{0}^{T}[u(t)-D w(t)] \mathrm{d} t=0,
$$

the latter implying that

$$
\lim _{T \rightarrow \infty} \frac{1}{T} \int_{0}^{T} u(t) \mathrm{d} t=\lim _{T \rightarrow \infty} \frac{1}{T} \int_{0}^{T} D w(t) \mathrm{d} t
$$

which is exactly condition (1.7). Finally, note that the average constraint can be generalized to $\lim _{T \rightarrow \infty} A v[w]=$ $\bar{w} \Rightarrow \lim _{T \rightarrow \infty} A v[u]=\bar{u}$, for any pair of nominal demand $\bar{w}$ and nominal controlled flow $\bar{u}$ such that $B \bar{u}=\bar{w}$. This can be done by simply translating the origin of the vector spaces of $w$ and $u$ in $\bar{w}$ and $\bar{u}$ respectively as we will show in the numerical example of Section 5.

\section{LOOKING FOR A GOOD OBJECTIVE FUNCTION $g$}

Here we outline the reasoning leading to the guess of a suitable objective function $g$. We recall that we are concerned only with what happens when $z(\cdot)$, the trajectory of $(0.2)$, is inside the target $\mathcal{T}$ for all the times.

Remark 2.1. Observe that problem has a trivial solution, for instance $g(\zeta, \mu, \omega)=\|\mu-\operatorname{sat}(-k \zeta)\|$. However, in this case the values $V$ and $\tilde{V}$ are constantly equal to zero, and so the disturbance $w(\cdot)$ does not play any effective role in selecting an optimal strategy by the first player. Instead, we are interested in a model that is suitable to model real situations in the applications (see Sect. 1). 
Since inside the target the saturated control is linear, we then look for a function $g$ with a relevant "quadratic feature". Moreover, we also ask that the corresponding value function (i.e. the optimum) is "easy to guess/construct", that is it has a relevant quadratic feature too.

First guess for $g$. A first natural choice for $g$ seems to be the following:

$$
g_{1}(\zeta, \mu, \omega)=\frac{k+1}{2}\left\|\zeta+\frac{D \omega}{k}\right\|^{2}+\frac{1}{2 k}\|\mu-D \omega\|^{2}
$$

However, such a choice does not force the demand to switch among only two vertices, which is instead what we would like to happen, otherwise the computation of the optima values may be harder (the case where the demand is free to switch among more than two vertices is still under investigation). Hence we suppose that there exists a vertex $\bar{\omega} \in \mathcal{W}$ such that

$$
\|D \bar{\omega}\|>\max _{\omega \in \mathcal{W}, \omega \neq \pm \bar{\omega}}\|D \omega\|
$$

This may happen, for instance, if the matrix $D$ has suitable coefficients (for example all positive), and such a requirement is compatible with real applications. However, there are other ways to impose the demand switch only among two opposite vertices. We then consider the following new objective function:

$$
g_{2}(\zeta, \mu, \omega)=\frac{k+1}{2}\left\|\zeta+\frac{D \omega}{k}\right\|^{2}+\frac{1}{2 k}\|\mu-D \omega\|^{2}+C_{1}\|D \omega\|^{2},
$$

where $C_{1}$ is a suitable positive constant. When the saturated control is used, the cost $g_{2}$ becomes

$$
g_{2}(\zeta,-k \zeta, \omega)=\frac{2 k+1}{2}\left\|\zeta+\frac{D \omega}{k}\right\|^{2}+C_{1}\|D \omega\|^{2} .
$$

When the demand is equal to $\bar{\omega}$, then $g_{2}$ in some sense weights the distance of $\zeta$ from $-(D \bar{\omega}) / k$, which is also the limit point of the trajectory with saturated linear control and fixed demand equal to $\bar{\omega}$. Hence a natural guess for the maximum/optimum would be

$$
V_{2}(\zeta)= \begin{cases}\frac{1}{2}\left\|\zeta+\frac{D \bar{\omega}}{k}\right\|^{2}+C_{1}\|D \bar{\omega}\|^{2} & \text { if } \zeta \cdot D \bar{\omega} \geq 0 \\ \frac{1}{2}\left\|\zeta-\frac{D \bar{\omega}}{k}\right\|^{2}+C_{1}\|D \bar{\omega}\|^{2} & \text { if } \zeta \cdot D \bar{\omega}<0\end{cases}
$$

However, such a function is not the good one, as it is easy to check since it does not solve the corresponding Hamilton-Jacobi equation (note that $V_{2}$ is continuous everywhere and of class $C^{1}$ out of the line $\zeta \cdot D \bar{\omega}=0$ ). Indeed, it seems that the optimal choice for $\omega$ is a sort of anticipation in the switching between $\bar{\omega}$ and $-\bar{\omega}$ before crossing the line $\zeta \cdot D \bar{\omega}$ (and note that, when using the saturated control $-k \zeta$ and the constant demand $\bar{\omega}$, the trajectory converges to the equilibrium point $-(D \bar{\omega}) / k$, and hence, starting from $\zeta_{0}$ with $\zeta_{0} \cdot D \bar{\omega}>0$ we certainly cross the line $\zeta \cdot D \bar{\omega}=0$ in a finite time). Hence, the value function for this maximization problem, has a more complicated formulation than $V_{2}$.

Second guess for $\mathrm{g}$. We introduce in the objective function a further term in order to force the maximizing demand to keep a constant value $(\bar{\omega}$ or $-\bar{\omega})$ when the state is inside one of the two parts $\zeta \cdot D \bar{\omega}>0$ or $\zeta \cdot D \bar{\omega}<0$. To this end, we modify the objective function in the following way

$$
g_{3}(\zeta, \mu, \omega)=\frac{k+1}{2}\left\|\zeta+\frac{D \omega}{k}\right\|^{2}+\frac{1}{2 k}\|\mu-D \omega\|^{2}+C_{1}\|D \omega\|^{2}+C_{2} \operatorname{sign}(\zeta \cdot D \omega),
$$


where $\operatorname{sign}$ is the $\operatorname{sign}$ function: $\operatorname{sign}(\xi)=1$ if $\xi \geq 0, \operatorname{sign}(\xi)=-1$ if $\xi<0$, and $C_{2}$ is a suitable nonzero constant not necessarily positive. In particular, if $C_{2}>0$ then the optimal choice for $\omega$ (when $\mu=-k \zeta$, the saturated control) should be $\bar{\omega}$ (respectively $-\bar{\omega}$ ) if $\zeta \cdot D \bar{\omega}>0$ (respectively $\zeta \cdot D \bar{\omega}<0$ ). Otherwise, if $C_{2}<0$, the optimal choice should be the opposite one. Unfortunately, the objective function $g_{3}$ becomes now discontinuous (since so is the sign function). This is a serious problem for implementing our procedure which is strongly based on uniqueness results of the Hamilton-Jacobi-Bellman equation for the maximization problem, as well as of the Hamilton-Jacobi-Isaacs equation for the differential game problem. In particular, note that the discontinuity of $g_{3}$ presents some new features which are not well studied in the literature. Indeed, such discontinuity is with respect (simultaneously) the state variables $\zeta$ as well as the control variables $\omega$, which are intrinsically related to each other by the scalar product $\zeta \cdot D \omega$.

Third guess for $g$. We approximate the sign function. One way may be to do that by continuous functions. However such a procedure, even if possible, will probably make us loose the "good fashion" of the value function $V_{2}$ which is almost based on the hypotheses that an optimal choice for the demand $\omega$ is switching through the line $\zeta \cdot D \bar{\omega}$. Hence, we prefer to approximate the sign function in a different way. We maintain the discontinuity but we introduce a sort of delay in the switching. This is done by replacing the sign function by the delayed thermostat $h_{\varepsilon}$ described in the Appendix. Let us denote by by $\varphi$ an odd function on $\mathcal{W}$ taking only the values 1 and -1 and such that $\varphi(\bar{\omega})=\operatorname{sign}\left(C_{2}\right)$. We then consider the objective function

$$
g_{4}(\zeta, \eta, \mu, \omega)=\frac{k+1}{2}\left\|\zeta+\frac{D \omega}{k}\right\|^{2}+\frac{1}{2 k}\|\mu-D \omega\|^{2}+C_{1}\|D \omega\|^{2}+C_{2} \eta \varphi(\omega)
$$

where the new state variable $\eta$ is subject to the evolution

$$
\eta(t)=h_{\varepsilon}\left[z(t) \cdot D \bar{\omega}, \eta_{0}\right](t)
$$

We have then introduced the new state variable $\eta$, which can only take the values 1 and -1 (the output of the thermostat) and whose evolution is subject to "logic rules", and not to "differential rules". That is we are considering a hybrid problem. Also note that $g_{4}$ is now continuous with respect to the state variables $\zeta$ and $\eta$. However note that the discontinuity in the state is not definitely disappeared. It is now present in the evolution of the variables $\eta$ which switches between two values. But such a switching is governed by the delayed thermostatic rule which allows us to say that switchings cannot accumulate in finite time (no Zeno phenomenon). Note that now we have two switching lines for $\eta$ : the line $\zeta \cdot D \bar{\omega}=\varepsilon$ only for switching up (from -1 to 1 ), and the line $\zeta \cdot D \bar{\omega}=-\varepsilon$ only for switching down (from 1 to -1 ). Unfortunately, even if the objective function $g_{4}$ seems good for the maximization problem in $\omega$ with the saturated control, it is no more suitable for the differential game problem. Indeed it may happen that, for particular values of $\zeta$ and $\eta$, the optimal choice of the minimization is to force the trajectory to cross one of the switching line, and to do that a different control from the linear saturated one may be necessary (recall that our final goal is to have the optimality of the saturated control, regardless of the behavior of the demand).

Fourth guess for $g$. The above outlined difficulty comes from the fact that the function "distance of $\zeta$ from $\pm(D \bar{\omega}) / k$ ", where the sign \pm depends on which the maximizing $\omega$ is, is not continuous through the switching lines $\zeta \cdot D \bar{\omega}= \pm \varepsilon$ (note that it is instead continuous through the switching line $\zeta \cdot D \bar{\omega}=0$, in the case of the sign function instead of the delayed relay). Hence we introduce a further term in the objective function which counts the number of switchings and, in some sense, makes that distance function continuous through the switching lines. The new objective function is (see Rem. 3.1 for the meaning of the constant $(2 \varepsilon) / k$ )

$$
g_{5}(\zeta, \eta, \sigma, \mu, \omega)=\frac{k+1}{2}\left\|\zeta+\frac{D \omega}{k}\right\|^{2}+\frac{1}{2 k}\|\mu-D \omega\|^{2}+C_{1}\|D \omega\|^{2}+C_{2} \eta \varphi(\omega)-\operatorname{sign}\left(C_{2}\right) \frac{2 \varepsilon}{k} \sigma,
$$




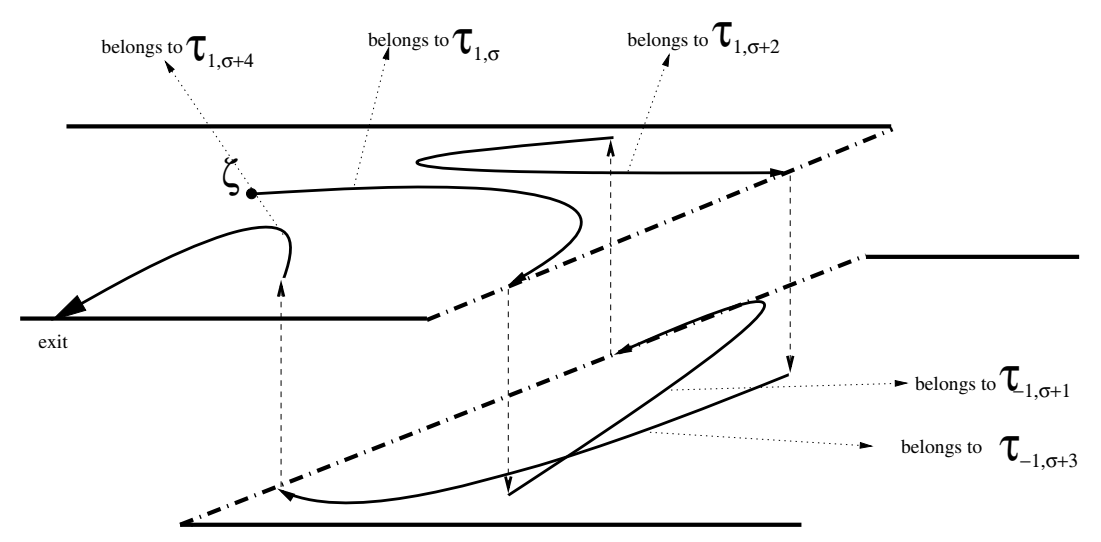

nonswitching boundary

. switching boundary

FIGURE 1. Switching evolution, starting from $\zeta$, which exits from the nonswitching boundary after four switchings.

where the new state variable $\sigma$ belongs to $\mathbb{N}$, and its discrete evolution is subject to a delayed switching rule given by (the term between parentheses can be expressed as the half of the total variation of $\eta$ in $[0, t]$ )

$$
\left.\sigma(t)=\sigma_{0}+\text { (number of switchings of } \eta \text { in }[0, t]\right) .
$$

\section{Formulation of the problems}

In the following, by $g(\zeta, \eta, \sigma, \mu, \omega)$ we intend the function $g_{5}$ as in $(2.5)$, and $\mathcal{T}$ is the target set defined in (0.6). We define the following sets for the state variables $(\zeta, \eta, \sigma) \in \mathcal{T} \times\{-1,1\} \times \mathbb{N}$. For every fixed $\sigma \in \mathbb{N}$ we define:

$$
\begin{aligned}
& \mathcal{T}_{1, \sigma}=\{(\zeta, 1, \sigma) \mid \zeta \in \mathcal{T}, \zeta \cdot D \bar{\omega} \geq-\varepsilon\}, \\
& \mathcal{T}_{-1, \sigma}=\{(\zeta,-1, \sigma) \mid \zeta \in \mathcal{T}, \zeta \cdot D \bar{\omega} \leq \varepsilon\}
\end{aligned}
$$

or, more generally, for every fixed $\eta \in\{-1,1\}$ and $\sigma \in \mathbb{N}$ :

$$
\mathcal{T}_{\eta, \sigma}=\{(\zeta, \eta, \sigma) \mid \zeta \in \mathcal{T}, \eta(\zeta \cdot D \bar{\omega}) \geq-\varepsilon\}
$$

For every initial state $\left(\zeta, \eta_{0}, \sigma_{0}\right) \in \mathcal{T}_{\eta_{0}, \sigma_{0}}$, the controlled dynamical system is

$$
\left\{\begin{array}{l}
z^{\prime}(t)=u(t)-D \omega(t) \\
\eta(t)=h_{\varepsilon}\left[z(\cdot) \cdot D \bar{\omega} ; \eta_{0}\right](t) \\
\sigma(t)=\sigma_{0}+(\text { number of switchings of } \eta \text { in }[0, t]), \\
z(0)=\zeta
\end{array}\right.
$$

We can say that $\mathcal{T}_{\eta, \sigma}$ is the set such that a trajectory of $(3.1)$, starting from $(\zeta, \eta, \sigma) \in \mathcal{T}_{\eta, \sigma}$, does not incur in any switching of $\eta(\cdot)$ and $\sigma(\cdot)$ (i.e. switching of the thermostat $h_{\varepsilon}$ ) until it remains inside $\mathcal{T}_{\eta, \sigma}$ (see Fig. 1). 
Note that, if we start from $\left(\zeta, \eta_{0}, \sigma_{0}\right) \in \mathcal{T}_{\eta_{0}, \sigma_{0}}$, and if the evolution of $z(\cdot)$ remains inside $\mathcal{T}$ for all the times, then we certainly have

$$
(z(t), \eta(t), \sigma(t)) \in \bigcup_{n \in \mathbb{N}} \mathcal{T}_{(-1)^{n} \eta_{0}, \sigma_{0}+n}
$$

Finally we consider the following exit-cost which acts when the trajectory $z(\cdot)$ leaves the closed target $\mathcal{T}$ (note that the evolution of $z(\cdot)$ is not subject to the evolution of $\eta$ and $\sigma$ ):

$$
\psi(\zeta, \eta, \sigma)=\frac{1}{2}\left\|\zeta+\operatorname{sign}\left(C_{2}\right) \eta \frac{D \bar{\omega}}{k}\right\|^{2}+C_{1}\|D \bar{\omega}\|^{2}+\left|C_{2}\right|-\operatorname{sign}\left(C_{2}\right) \frac{2 \varepsilon}{k} \sigma .
$$

Remark 3.1. Note that, since the dynamics $\mu-D \omega$ is bounded, then, for passing from one switching line to the other, it is necessary to spend at least a time $\tau>0$ independently from the initial position on one of the two switching lines and from the controls $\mu$ and $\omega$. Moreover, in that case of the saturated control $\mu(\cdot)=-k z(\cdot)$, for every initial state $\left(\zeta, \eta_{0}, \sigma_{0}\right)$ there exists a sequence of controls $w_{\delta}(\cdot)$ for the demand such that the corresponding costs converge to $\psi\left(\zeta, \eta_{0}, \sigma_{0}\right)$, as $\delta \rightarrow 0^{+}$. For instance, if $C_{2}>0$, let us fix $\delta>0$ and start with $w \equiv \eta_{0} \bar{\omega}$ until $t_{1}+\delta$ where $t_{1}$ is the first switching instant for the trajectory $z(\cdot)$ (it coincides with the reaching time of the switching line $\zeta \cdot D \bar{\omega}=-\eta_{0} \varepsilon$, which happens in a finite time). At the instant $t_{1}$ the trajectory $(\eta, \sigma)$ switches and then we let $w$ switch at the instant $t_{1}+\delta$ positioning on $-\eta_{0} \bar{\omega}$. Hence, the trajectory $z(\cdot)$ changes direction, and the system goes on in this way for all the time. At every switching instant, the objective function $g(z(t), \eta(t), \sigma(t),-k z(t), \eta(t) \bar{\omega})$ has a decrement of the quantity $-(2 \varepsilon) / k$ in its term containing the switching variable $\sigma$ which, via integration in the time up to $+\infty$, is exactly compensated (modulo a quantity which goes to zero as $\delta$ goes to zero) by the increment in the quadratic part $\left((2 k+1)\|z(t)+\eta(t) D \bar{\omega} / k\|^{2}\right) / 2(-(2 \varepsilon) / k$ is equal to half of the difference between the square distances of a point on the switching line from the two points $\pm(D \bar{\omega}) / k)$. Hence, just a direct calculation proves the guess.

\subsection{Maximization problem}

When the control $u \in U$ is fixed equal to the linear saturated control $-k z(\cdot)$, we have the maximization problem in $w \in W$ given by the exit-time problem:

$$
\begin{aligned}
V(\zeta, \eta, \sigma)= & \max _{w \in W} \int_{0}^{t} \mathrm{e}^{-s} g(z(s), \eta(s), \sigma(s),-k z(s), w(s)) \mathrm{d} s \\
& +\mathrm{e}^{-t} \psi(z(t), \eta(t), \sigma(t)), \quad t=t_{\zeta, w}
\end{aligned}
$$

where $t_{\zeta, w}$ is the first exit time of $z(\cdot)$ from the target $\mathcal{T}$, under the demand $w$. Since there exists a minimum time interval in order to pass from one switching line to the other (see Rem. 3.1), then, starting from any point $(\zeta, \eta, \sigma)$, with any controls, the cost is bounded (i.e. the integral converges, even if the trajectory $z(\cdot)$ never exits from $\mathcal{T}$ ). Indeed, what may be not bounded in $g$ is the variable $\sigma$ which, if we have infinitely many switchings, goes to $+\infty$. However, due to the uniform delay in time for every possible switching, and due to the presence of the discount exponential factor $\mathrm{e}^{-t}$, the integral converges anyway.

Let us denote by int $\mathcal{T}$ and by $\partial \mathcal{T}$ respectively the interior and the boundary of $\mathcal{T}$ (as subset of $\mathbb{R}^{n}$ ). For every $(\eta, \sigma) \in\{-1,1\} \times \mathbb{N}$ fixed we denote the interior of $\mathcal{T}_{\eta, \sigma}$ ("interior" with respect to $\zeta$ as subset of $\mathbb{R} \times\{\eta\} \times\{\sigma\}$ )

$$
\left.\operatorname{int} \mathcal{T}_{\eta, \sigma}=\left\{(\zeta, \eta, \sigma) \mid(\zeta, \eta, \sigma) \in \mathcal{T}_{\eta, \sigma}, \zeta \in \operatorname{int} \mathcal{T}, \eta(\zeta \cdot D \bar{\omega})>-\varepsilon\right)\right\}
$$

For every $(\eta, \sigma) \in\{-1,1\} \times \mathbb{N}$, the boundary of $\mathcal{T}_{\eta, \sigma}$ ("boundary" with respect to $\zeta$ as subset of $\mathbb{R} \times\{\eta\} \times\{\sigma\}$ ) is split in two parts (nonswitching boundary and switching boundary respectively, see the figure)

$$
\begin{aligned}
& \partial \mathcal{T}_{\eta, \sigma}^{n s}=\left\{(\zeta, \eta, \sigma) \mid(\zeta, \eta, \sigma) \in \mathcal{T}_{\eta, \sigma}, \zeta \in \partial \mathcal{T}, \eta(\zeta \cdot D \bar{\omega})>-\varepsilon\right\}, \\
& \partial \mathcal{T}_{\eta, \sigma}^{s}=\left\{(\zeta, \eta, \sigma) \mid(\zeta, \eta, \sigma) \in \mathcal{T}_{\eta, \sigma}, \eta(\zeta \cdot D \bar{\omega})=-\varepsilon\right\} .
\end{aligned}
$$


Finally, we define

$$
\hat{\mathcal{T}}=\bigcup_{n \in \mathbb{N}} \mathcal{T}_{1, n} \cup \bigcup_{n \in \mathbb{N}} \mathcal{T}_{-1, n}
$$

Since the delayed thermostat satisfies a suitable "semigroup property" (see Visintin [25]), the Dynamic Programming Principle holds: for every $(\zeta, \eta, \sigma) \in \hat{\mathcal{T}}$, and for every $t>0$ we have

$$
V(\zeta, \eta, \sigma)=\sup _{w \in W}\left(\int_{0}^{\bar{t}} \mathrm{e}^{-s} g(z(s), \eta(s), \sigma(s),-k z(s), w(s)) \mathrm{d} s+\mathrm{e}^{-\bar{t}} V(z(\bar{t}), \eta(\bar{t}), \sigma(\bar{t}))\right),
$$

where $\bar{t}=\min \left(t, t_{\zeta, w}\right)$.

Proposition 3.2. The value function $V$ is continuous on $\hat{\mathcal{T}}$, i.e. separately on every $\mathcal{T}_{\eta, \sigma}$.

Proof. Note that, for $\varepsilon$ sufficiently small, the points on the switching lines are totally controllable. Indeed, for instance, the normal to the line $\zeta \cdot D \bar{\omega}=\varepsilon$ is of course given by $D \bar{\omega}$, and, if $\zeta$ is on that line, we have

$$
(-k \zeta-D \bar{\omega}) \cdot D \bar{\omega}<0<(-k \zeta+D \bar{\omega}) \cdot D \bar{\omega}
$$

where $-k \zeta-D \bar{\omega}$, and $-k \zeta+D \bar{\omega}$ are the two possible dynamics. Moreover, for every initial point $(\zeta, \eta, \sigma) \in \partial \mathcal{T}_{\eta, \sigma}^{n s}$, all possible choice of $\omega$ make the trajectory strictly entering in $\operatorname{int} \mathcal{T}_{\eta, \varepsilon}$. Hence, the trajectory cannot exit from $\mathcal{T}_{\eta, \sigma}$ through $\partial \mathcal{T}_{\eta, \sigma}^{n s}$, and hence the exit-cost $\psi$ does not play a true role. The trajectory may exit from $\mathcal{T}_{\eta, \sigma}$ only through the switching boundary $\partial \mathcal{T}_{\eta, \sigma}^{s}$. That is $t_{\zeta, \omega}=+\infty$ for all $\zeta \in \mathcal{T}$ and $\omega$. Hence, the only fact that may cause discontinuity is the presence of the two switching lines $\zeta \cdot D \bar{\omega}=\varepsilon$ and $\zeta \cdot D \bar{\omega}=-\varepsilon$ (which act as switching line only if $\eta(t)=-1$ and $\eta(t)=1$ respectively). However, the total (inward/outward) controllability on that lines and the fact that two possible subsequent switching time are delayed at least by an independent positive time make $V$ continuous anyway, as can be proved by standard techniques.

For every $(\eta, \sigma) \in\{-1,1\} \times \mathbb{N}$, we consider the following Hamiltonian in $\mathbb{R}^{n} \times \mathbb{R}^{n}$

$$
H_{\eta, \sigma}(\zeta, p)=\min _{\omega \in \mathcal{W}}\{-(-k \zeta-D \omega) \cdot p-g(\zeta, \eta, \sigma,-k \zeta, \omega)\} .
$$

For every $(\eta, \sigma) \in\{-1,1\} \times \mathbb{N}$, we consider the following Hamilton-Jacobi-Bellman problem in $\mathcal{T}_{\eta, \sigma}$ with (partial) boundary condition, which, for a generic solution $(\zeta, \eta, \sigma) \mapsto v(\zeta, \eta, \sigma)$ (which of course depends only on $\zeta$, since $\eta$ and $\sigma$ are fixed in $\mathcal{T}_{\eta, \sigma}$ ), is written as

$$
\begin{cases}v(\zeta, \eta, \sigma)+H_{\eta, \sigma}\left(\zeta, \nabla_{\zeta} v(\zeta, \eta, \sigma)\right)=0 & \text { in } \operatorname{int} \mathcal{T}_{\eta, \sigma} \\ v(\zeta, \eta, \sigma)=\psi(\zeta, \eta, \sigma) & \text { on } \partial \mathcal{T}_{\eta, \sigma}^{n s}\end{cases}
$$

where $\nabla_{\zeta}$ means the gradient with respect to $\zeta$ only. Note that the boundary condition is "partial" since it is imposed only on the nonswitching boundary $\partial \mathcal{T}_{\eta, \sigma}^{n s}$. We call (3.4) $H J B_{\eta, \sigma}$.

Proposition 3.3. Standing all the hypotheses already assumed, the value function $V$ of our maximization problem is the unique continuous function on $\hat{\mathcal{T}}$ satisfying both

$$
\begin{gathered}
V(\zeta, \eta, \sigma)-V(\zeta, \eta, \sigma+1)=-\operatorname{sign}\left(C_{2}\right) \frac{2 \varepsilon}{k} \\
V(\zeta, \eta, \sigma) \geq \max \{\psi(\zeta, \eta, \sigma), V(\zeta,-\eta, \sigma+1)\} \quad \forall(\zeta, \eta, \sigma) \in \partial \mathcal{T}_{\eta, \sigma}^{s} \cap \partial \mathcal{T}_{\eta, \sigma}^{n s},
\end{gathered}
$$


and finally which solves the following problem

$$
\left\{\begin{array}{l}
\forall(\eta, \sigma) \in\{-1,1\} \times \mathbb{N}, V \text { solves the following in the viscosity sense: } \\
\left\{\begin{array}{l}
V \text { solves } H J B_{\eta, \sigma}(3.4), \\
V(\zeta, \eta, \sigma)=V(\zeta,-\eta, \sigma+1) \text { on } \partial \mathcal{T}_{\eta, \sigma}^{s} .
\end{array}\right.
\end{array}\right.
$$

Remark 3.4. We refer to Proposition 6.1 , regarding $\mathcal{T}_{\eta, \sigma}$ as $\bar{\Omega}, \partial \mathcal{T}_{\eta, \sigma}^{s}$ as $(\partial \Omega)_{1}$, and $\partial \mathcal{T}_{\eta, \sigma}^{n s}$ as $(\partial \Omega)_{\hat{1}}$. By virtue of (3.3), which in our case also implies that the analogous of $(6.9)$ holds, for every $(\eta, \sigma)$ fixed and for every continuous function $h$ defined on $\partial \mathcal{T}_{\eta, \sigma}^{s}$, satisfying

$$
h(\zeta, \eta, \sigma) \geq \psi(\zeta, \eta, \sigma) \text { on } \partial \mathcal{T}_{\eta, \sigma}^{s} \cap \partial \mathcal{T}_{\eta, \sigma}^{n s}
$$

there exists a unique continuous function $v$ defined on $\mathcal{T}_{\eta, \sigma}$ which solves the problem

$$
\left\{\begin{array}{l}
v \text { solves } H J B_{\eta, \sigma}(3.4) \\
v(\zeta, \eta, \sigma)=h(\zeta, \eta, \sigma) \text { on } \partial \mathcal{T}_{\eta, \sigma}^{s}
\end{array}\right.
$$

and it coincides with the value function of the maximization problem in $\mathcal{T}_{\eta, \sigma}$ with exit cost given by $\psi$ on $\partial \mathcal{T}_{\eta, \sigma}^{n s}$ (which does not play any role by (6.8)) and by $h$ on $\partial \mathcal{T}_{\eta, \sigma}^{s}$. However, this fact does not immediately implies the uniqueness for the Hamilton-Jacobi problem as in the proposition above, since there the boundary conditions (i.e. the exit-costs) are mutually exchanged on the switching boundaries, i.e. they are part of the solution. However, such a uniqueness result for every $(\eta, \sigma)$ fixed will be of course used in the proof.

Proof of Proposition 3.3. By Proposition 3.2, the value function $V$ of our maximization problem is continuous on $\hat{\mathcal{T}}$. Moreover it also satisfies (3.5). Indeed, all the initial states $(\zeta, \eta, \sigma+n)$, with $n \in \mathbb{N}$, are exactly in the same situation with respect to the future evolution and to the cost $g$, except for the value $-\operatorname{sign}\left(C_{2}\right)(2 \varepsilon) / k$.

It solves (3.6) because, by definition of $\psi$, by the argumentation of Remark 3.1, and by the controllability (6.7) we have that for all $(\zeta, \eta, \sigma) \in \partial \mathcal{T}_{\eta, \sigma}^{s} \cap \partial \mathcal{T}_{\eta, \sigma}^{n s}$

$$
\begin{aligned}
& V(\zeta, \eta, \sigma) \geq V(\zeta,-\eta, \sigma+1) \\
& V(\zeta,-\eta, \sigma+1) \geq \psi(\zeta,-\eta, \sigma+1)=\psi(\zeta, \eta, \sigma)
\end{aligned}
$$

It also solves (3.7). Indeed, for every $(\zeta, \eta, \sigma) \in \mathcal{T}_{\eta, \sigma}$ and for every control $w \in W$ let us denote by $t^{w} \leq$ $+\infty$ the corresponding exit-time from $\mathcal{T}_{\eta, \sigma}$ (recall that the exit time of $z(\cdot)$ from $\mathcal{T}$ is always equal to $+\infty$, $\left.t_{\zeta}, w=+\infty\right)$. Let us fix $(\zeta, \eta, \sigma) \in \mathcal{T}_{\eta, \sigma}$, and suppose that, for every $\varepsilon>0$ there exists a control $w_{\varepsilon}$ which is $\varepsilon$-optimal and such that $t^{w_{\varepsilon}}$ is finite (if not, the case is easier). Then, for $\delta>0$ sufficiently small, we have (using the definition of $V$, the inequality (3.8) and the Dynamic Programming Principle (3.2), also recall that, by definition of the switching rule for the delayed thermostat, at the switching instant $t^{w}$ the switching is not yet happened)

$$
\begin{aligned}
\int_{0}^{t^{w_{\varepsilon}}+\delta} \mathrm{e}^{-s} g(z(s), \eta(s), \sigma(s) & \left.,-k z(s), w_{\varepsilon}(s)\right) \mathrm{d} s+\mathrm{e}^{-t^{w_{\varepsilon}}} V\left(z\left(t^{w_{\varepsilon}}+\delta\right),-\eta, \sigma+1\right)+\varepsilon \geq V(\zeta, \eta, \sigma) \\
& \geq \sup _{w \in W}\left(\int_{0}^{t^{w}} \mathrm{e}^{-s} g(z(s), \eta, \sigma,-k z(s), w(s)) \mathrm{d} s+\mathrm{e}^{-t^{w}} V\left(z\left(t^{w}\right), \eta, \sigma\right)\right) \\
& \geq \sup _{w \in W}\left(\int_{0}^{t^{w}} \mathrm{e}^{-s} g(z(s), \eta, \sigma,-k z(s), w(s)) \mathrm{d} s+\mathrm{e}^{-t^{w}} V\left(z\left(t^{w}\right),-\eta, \sigma+1\right)\right) .
\end{aligned}
$$


Taking the limit $\delta \rightarrow 0^{+}$, using the continuity of $V$ and the arbitrariness of $\varepsilon>0$ we get, for every $(\zeta, \eta, \sigma) \in \mathcal{T}_{\eta, \sigma}$

$$
V(\zeta, \eta, \sigma)=\sup _{w \in W}\left(\int_{0}^{t^{w}} \mathrm{e}^{-s} g(z(s), \eta, \sigma,-k z(s), w(s)) \mathrm{d} s+\mathrm{e}^{-t_{w}} V\left(z\left(t^{w}\right),-\eta, \sigma+1\right)\right) .
$$

This means that on every $\mathcal{T}_{\eta, \sigma}, V$ is the value function of the maximization problem with exit cost from $\mathcal{T}_{\eta, \sigma}$ given by $\psi$ on $\partial \mathcal{T}_{\eta, \sigma}^{n s}$ (which, however, does not play any role) and by $V(\cdot,-\eta, \sigma+1)$ on $\partial \mathcal{T}_{\eta, \sigma}^{s}$. Hence, by Remark 3.4, $V$ satisfies the Hamilton-Jacobi problem (3.7).

Now let us prove the uniqueness. Let us consider two continuous functions $f, h$ defined on $\hat{\mathcal{T}}$ which satisfy the same inequalities (3.5), (3.6) satisfied by $V$, and the Hamilton-Jacobi problem (3.7). We are going to prove that $f=h$. For every $(\eta, \sigma)$ fixed, $f$ and $h$ are, on $\mathcal{T}_{\eta, \sigma}$ (see Rem. 3.4), the value function of two exit-time maximization problems which differs from each other only for the exit-costs on the switching boundary $\partial \mathcal{T}_{\eta, \sigma}^{s}$. Those two exit-costs are given respectively by $f(\zeta,-\eta, \sigma+1)$ and $h(\zeta,-\eta, \sigma+1)$. The same argumentation shows that, on $\mathcal{T}_{-\eta, \sigma+1} f$ and $h$ are the value functions of two exit-time maximization problems which differ from each other only for the exit costs on the switching boundary $\partial \mathcal{T}_{-\eta, \sigma+1}^{s}$. Now, recalling that we need to spend at least an independent time $\tau>0$ in order to pass from one switching boundary to the other, and recalling $(3.5)$ we get, for every fixed $(\eta, \sigma)$

$$
\begin{aligned}
\sup _{(\zeta, \eta, \sigma) \in \mathcal{T}_{\eta, \sigma}}|f(\zeta, \eta, \sigma)-h(\zeta, \eta, \sigma)| & \leq \sup _{(\zeta, \eta, \sigma) \in \partial \mathcal{T}_{\eta, \sigma}^{s}}|f(\zeta,-\eta, \sigma+1)-h(\zeta,-\eta, \sigma+1)| \\
& \leq \mathrm{e}^{-\tau} \sup _{(\zeta,-\eta, \sigma+1) \in \partial \mathcal{T}_{-\eta, \sigma+1}^{s}}|f(\zeta, \eta, \sigma+2)-h(\zeta, \eta, \sigma+2)| \\
& \leq \mathrm{e}^{-\tau} \sup _{(\zeta, \eta, \sigma+2) \in \mathcal{T}_{\eta, \sigma+2}}|f(\zeta, \eta, \sigma+2)-h(\zeta, \eta, \sigma+2)| \\
& =\mathrm{e}^{-\tau} \sup _{(\zeta, \eta, \sigma) \in \mathcal{T}_{\eta, \sigma}}|f(\zeta, \eta, \sigma)-h(\zeta, \eta, \sigma)|
\end{aligned}
$$

from which the desired result since $0<\mathrm{e}^{-\tau}<1$.

\subsection{The MinMax problem}

Here the problem is given by the (lower) value function

$$
\begin{aligned}
\tilde{V}(\zeta, \eta, \sigma)= & \inf _{\gamma \in \Gamma} \sup _{w \in W} \int_{0}^{\tilde{t}} \mathrm{e}^{-s} g(z(s), \eta(s), \sigma(s), \gamma[w](s), w(s)) \mathrm{d} s \\
& +\mathrm{e}^{-\tilde{t}} \psi(z(\tilde{t}), \eta(\tilde{t}), \sigma(\tilde{t})), \quad \tilde{t}=t_{\zeta, \gamma, w}
\end{aligned}
$$

where $\Gamma$ is the set of non-anticipative strategies for the first player (the minimizing one), and $t_{\zeta, \gamma, w} \leq+\infty$ is the first exit time of $z(\cdot)$ from the closed of the target $\mathcal{T}$ under the demand $w$ and the strategy $\gamma$.

Also in this case, the Dynamic Programming Principle holds: for every $(\zeta, \eta, \sigma) \in \hat{\mathcal{T}}$, and for every $t>0$ we have

$$
\tilde{V}(\zeta, \eta, \sigma)=\inf _{\gamma \in \Gamma} \sup _{w \in W}\left(\int_{0}^{\bar{t}} \mathrm{e}^{-s} g(z(s), \eta(s), \sigma(s), \gamma[w](s), w(s)) \mathrm{d} s+\mathrm{e}^{-\bar{t}} \tilde{V}(z(\bar{t}), \eta(\bar{t}), \sigma(\bar{t}))\right),
$$

where $\bar{t}=\min \left(t, t_{\zeta, \gamma, w}\right)$. 
Proposition 3.5. The value function $\tilde{V}$ is continuous in $\hat{\mathcal{T}}$.

Proof. Note that, by (0.5), the dynamical system $\dot{z}=u-D w$ is completely controllable in $z$ by the first player, whichever is the control $\omega$ used by the second player. That is, for every $(\zeta, \eta, \sigma) \in \partial \mathcal{T}_{\eta, \sigma}^{n s} \cup \partial \mathcal{T}_{\eta, \sigma}^{s}$, there exists two controls $\mu_{1}, \mu_{2} \in \mathcal{U}$ such that for every $\omega \in W$ the field $\mu_{1}-D \omega$, when applied in $\zeta$, is strictly inward in $\operatorname{int} \mathcal{T}_{\eta, \sigma}$ (i.e. $\left(\zeta+\nu\left(\mu_{1}-D \omega\right), \eta, \sigma\right) \in \operatorname{int} \mathcal{T}_{\eta, \sigma}$ for $\nu>0$ sufficiently small), and the field $\mu_{2}-D \omega$, when applied in $\zeta$, is strictly outward from $\mathcal{T}_{\eta, \sigma}\left(i . e .\left(\zeta+\nu\left(\mu_{2}-D \omega\right), \eta, \sigma\right) \notin \mathcal{T}_{\eta, \sigma}\right.$ for $\left.\nu>0\right)$.

Such a total controllability on the boundary and on the switching lines, together with the delay property of the switching instants, make the value function $\tilde{V}$ continuous, as can be proved by techniques similar to those of the proof of Proposition 6.2.

For every $(\eta, \sigma)$, we have the Hamiltonian, defined in $\mathbb{R}^{n} \times \mathbb{R}^{n}$,

$$
\tilde{H}_{\eta, \sigma}(\zeta, p)=\min _{\omega \in \mathcal{W}} \max _{\mu \in \mathcal{U}}\{-(\mu-D \omega) \cdot p-g(\zeta, \eta, \sigma, \mu, \omega)\}
$$

For every $(\eta, \sigma) \in\{-1,1\} \times \mathbb{N}$, we consider the Hamilton-Jacobi-Isaacs problem in $\mathcal{T}_{\eta \sigma}$ with (partial) boundary condition which, for a generic solution $(\zeta, \eta, \sigma) \mapsto v(\zeta, \eta, \sigma)$ (which of course depends only on $\zeta$, since $\eta$ and $\sigma$ are fixed in $\mathcal{T}_{\eta, \sigma}$ ), is written as

$$
\begin{cases}v(\zeta, \eta, \sigma)+\tilde{H}_{\eta, \sigma}\left(\zeta, \nabla_{\zeta} v(\zeta, \eta, \sigma)\right)=0 & \text { in } \mathcal{T}_{\eta, \sigma} \\ v(\zeta, \eta, \sigma)=\psi(\zeta, \eta, \sigma) & \text { on } \partial \mathcal{T}_{\eta, \sigma}^{n s}\end{cases}
$$

where $\nabla_{\zeta}$ means the gradient with respect to $\zeta$ only. Note that the boundary condition is "partial" since it is imposed only on the nonswitching boundary of $\mathcal{T}_{\eta, \sigma}$. We call $(3.10) \hat{H J} I_{\eta, \sigma}$.

Proposition 3.6. Standing all the hypotheses already assumed, the value function $\tilde{V}$ of our minmax problem is the unique continuous function on $\hat{\mathcal{T}}$ which satisfies (3.5) and the following inequality

$$
\tilde{V}(\zeta, \eta, \sigma) \leq \min \{\psi(\zeta, \eta, \sigma), \tilde{V}(\zeta,-\eta, \sigma+1)\} \quad \forall(\zeta, \eta, \sigma) \in \partial \mathcal{T}_{\eta, \sigma}^{s} \cap \partial \mathcal{T}_{\eta, \sigma}^{n s},
$$

and finally which solves the following problem

$$
\left\{\begin{array}{l}
\forall(\eta, \sigma) \in\{-1,1\} \times \mathbb{N}, \\
\tilde{V} \text { solves the following in the viscosity sense: } \\
\left\{\begin{array}{l}
\tilde{V} \text { solves } \tilde{H J} I_{\eta, \sigma}(3.10), \\
\tilde{V}(\zeta, \eta, \sigma)=\tilde{V}(\zeta,-\eta, \sigma+1) \text { on } \partial \mathcal{T}_{\eta, \sigma}^{s} .
\end{array}\right.
\end{array}\right.
$$

Remark 3.7. First, let us note that, for every $(\eta, \sigma)$ fixed, the boundary condition in $\tilde{H J I} I_{\eta, \sigma}(3.10)$ is now playing a true role (despite to what happens for the maximization problem) since, on the nonswitching boundary $\partial \mathcal{T}_{\eta, \sigma}^{n s}$, there are strategies for the first player that make the trajectory exit (by virtue of $\left.(0.5)\right)$. Moreover, (0.5) also implies that the point on $\partial \mathcal{T}_{\eta, \sigma}^{s} \cap \partial \mathcal{T}_{\eta, \sigma}^{n s}$ are totally controllable by the first player, and also that there exist strategies for the first player such the analogous of (6.16) holds. Hence, by Proposition 6.2 , for every $(\eta, \sigma)$ fixed, and for continuous function $h$ defined on the switching boundary $\partial \mathcal{T}_{\eta, \sigma}^{s}$, such that

$$
h(\zeta, \eta, \sigma) \leq \psi(\zeta, \eta, \sigma) \text { on } \partial \mathcal{T}_{\eta, \sigma}^{s} \cap \partial \mathcal{T}_{\eta, \sigma}^{n s}
$$

there exists a unique continuous function $\tilde{v}$ defined on $\mathcal{T}_{\eta, \sigma}$ such that

$$
\tilde{v}(\zeta, \eta, \sigma) \leq \min \{\psi(\zeta, \eta, \sigma), h(\zeta, \eta, \sigma)\} \text { on } \partial \mathcal{T}_{\eta, \sigma}^{s} \cap \partial \mathcal{T}_{\eta, \sigma}^{n s}
$$


and such that solves the problem

$$
\left\{\begin{array}{l}
\tilde{v} \text { solves } H J I_{\eta, \sigma}(3.10) \\
\tilde{v}(\zeta, \eta, \sigma)=h(\zeta, \eta, \sigma) \text { on } \partial \mathcal{T}_{\eta, \sigma}^{s}
\end{array}\right.
$$

Such a unique function $\tilde{v}$ coincides with the value function of the minmax problem in $\mathcal{T}_{\eta, \sigma}$ with exit cost given by $\psi$ on $\partial \mathcal{T}_{\eta, \sigma}^{n s}$ and by $h$ on $\partial \mathcal{T}_{\eta, \sigma}^{s}$. Final considerations as in Remark 3.4 then follow.

Proof of Proposition 3.6. Let us note that, by the controllability on the boundary, and by the definition of $\psi$, also in this case $\tilde{V}$ satisfies (3.11). Moreover, also in this case $\tilde{V}$ satisfies (3.5). The fact that $\tilde{V}$ satisfies (3.12), and that it is the unique solution comes, via Remark 3.7, by the same argumentation as in the proof of Proposition 3.3. In particular, using the Dynamic Programming Principle (3.9), suitably arguing as for the maximization problem, we have, for every $(\zeta, \eta, \sigma) \in \partial \mathcal{T}_{\eta, \sigma}^{s} \cap \partial \mathcal{T}_{\eta, \sigma}^{n s}$ (here $t^{\gamma, w}$ is the first switching instant of the trajectory)

$$
\begin{aligned}
& \sup _{w \in W}\left(\int_{0}^{t^{\gamma_{\varepsilon}, w}+\delta} \mathrm{e}^{-} s g\left(z(s), \eta(s), \sigma(s), \gamma_{\varepsilon}[w](s), w(s)\right) \mathrm{d} s+\mathrm{e}^{-t^{\gamma_{\varepsilon}, w}+\delta} \tilde{V}\left(z\left(t^{\gamma_{\varepsilon}, w}+\delta\right),-\eta, \sigma+1\right)\right)-\varepsilon \leq \tilde{V}(\zeta, \eta, \sigma) \\
& \leq \inf _{\gamma \in \Gamma} \sup _{w \in W}\left(\int_{0}^{t^{\gamma, w}} \mathrm{e}^{-} s g(z(s), \eta(s), \sigma(s), \gamma[w](s), w(s)) \mathrm{d} s+\mathrm{e}^{-t^{\gamma, w}} \tilde{V}\left(z\left(t^{\gamma, w}\right), \eta, \sigma\right)\right) \\
& \leq \inf _{\gamma \in \Gamma} \sup _{w \in W}\left(\int_{0}^{t^{\gamma, w}} \mathrm{e}^{-} s g(z(s), \eta(s), \sigma(s), \gamma[w](s), w(s)) \mathrm{d} s+\mathrm{e}^{-t^{\gamma, w}} \tilde{V}\left(z\left(t^{\gamma, w}\right),-\eta, \sigma+1\right)\right) \cdot
\end{aligned}
$$

\section{Robustness}

In this section, we proof the robustness of the linear saturated control in the sense of Definition 0.1. This is done exhibiting a continuous function on $\hat{\mathcal{T}}$ which solves both problems (3.7) and (3.12).

Proposition 4.1. For suitable choices of the constants $C_{1}$ and $C_{2}$, the function

$$
\phi(\zeta, \eta, \sigma)=\frac{1}{2}\left\|\zeta+\operatorname{sign}\left(C_{2}\right) \eta \frac{D \bar{\omega}}{k}\right\|^{2}+C_{1}\|D \bar{\omega}\|^{2}+\left|C_{2}\right|-\operatorname{sign}\left(C_{2}\right) \frac{2 \varepsilon}{k} \sigma,
$$

solves both problems (3.7) and (3.12).

Proof. It is evident that $\phi$ is continuous and that it satisfies (3.5), (3.6) and (3.11). Moreover, also the boundary condition are satisfied (even in the classical sense: point by point). Now, let us note that, for every $(\eta, \sigma)$ fixed, $\phi$ is of class $C^{1}$ (with respect to $\zeta$ ) in $\operatorname{int} \mathcal{T}_{\eta, \sigma}$, and that its gradient is

$$
\nabla_{\zeta} \phi(\zeta, \eta, \sigma)=\zeta+\operatorname{sign}\left(C_{2}\right) \eta \frac{D \bar{\omega}}{k}
$$

Hence, we have to find $C_{1}>0$ and $C_{2} \in \mathbb{R}$ such that, for every $(\eta, \sigma)$, the following holds in the classical sense:

$$
\begin{aligned}
& \phi(\zeta, \eta, \sigma)+H_{\eta, \sigma}\left(\zeta, \nabla_{\zeta} \phi(\zeta, \eta, \sigma)\right)=0, \quad \operatorname{in} \operatorname{int} \mathcal{T}_{\eta, \sigma} \\
& \phi(\zeta, \eta, \sigma)+\tilde{H}_{\eta, \sigma}\left(\zeta, \nabla_{\zeta} \phi(\zeta, \eta, \sigma)\right)=0, \quad \operatorname{in} \operatorname{int} \mathcal{T}_{\eta, \sigma} .
\end{aligned}
$$

By (2.1), by the compactness of $\mathcal{T}, \mathcal{U}$ and $\mathcal{W}$, the values of $C_{1}>0$ and of $\left|C_{2}\right|$ are easily constructed (recall also the definition of $\varphi$ which appears in the objective function $\left.g=g_{5}(2.5)\right)$. For instance, we can take, without any pretension of sharpness

$$
\begin{aligned}
& \left|C_{2}\right| \geq 2 \max _{\zeta \in \mathcal{T}, \mu \in \mathcal{U}, \omega \in \mathcal{W}, \eta \in\{-1,1\}}|f(\zeta, \eta, \mu, \omega)|, \\
& C_{1} \geq \max _{\zeta \in \mathcal{T}, \mu \in \mathcal{U}, \omega \in \operatorname{vert} \mathcal{W}, \omega \neq \pm \bar{\omega}, \eta \in\{-1,1\}} \frac{f(\zeta, \eta, \mu, \pm \bar{\omega})-f(\zeta, \mu, \omega)}{\|D \bar{\omega}\|^{2}-\|D \omega\|^{2}},
\end{aligned}
$$


where vert $\mathcal{W}$ is the set of the vertices of $\mathcal{W}$ and

$$
f(\zeta, \eta, \omega, \omega)=-(\mu-D \omega) \cdot\left(\zeta+\eta \frac{D \bar{\omega}}{k}\right)-\frac{k+1}{2}\left\|\zeta+\frac{D \omega}{k}\right\|^{2}-\frac{1}{2 k}\|\mu-D \omega\|^{2} .
$$

Proposition 4.2. If $C_{1}$ and $C_{2}$ are the ones outlined in Proposition 4.1, then the linear saturated control is robustly optimal in the sense of Definition 0.1 .

Proof. Given Proposition 4.1 and the uniqueness results of the previous section, the conclusion immediately follows, since we have $V=\tilde{V}=\phi$.

Remark 4.3. When $\varepsilon$ goes to zero, the thermostat $h_{\varepsilon}$ tends to the sign function. Moreover, the value function $V$ and $\tilde{V}$ (which are equal by the way), on every $\mathcal{T}_{\eta, \sigma}$, uniformly converge to the function

$$
V_{0}(\zeta, \eta)=\frac{1}{2}\left\|\zeta+\operatorname{sign}\left(C_{2}\right) \eta \frac{D \bar{\omega}}{k}\right\|^{2}+C_{1}\|D \bar{\omega}\|^{2}+\left|C_{2}\right|,
$$

which does not depend on $\sigma$ anymore, and with $\eta=\operatorname{sign}(\zeta \cdot D \bar{\omega})$. One may ask whether $V_{0}$ is the value for both the maximization problem and the minmax problem with the thermostat replaced by the sign function (something as in the "Second guess" paragraph). Unfortunately this is not true. A probably correct guess is that the almost optimal trajectories for the problems with the thermostat $h_{\varepsilon}$ (which, for the case $C_{2}>0$, are the ones described in Rem. 3.1) are also almost optimal for the limit problems with the sign function. This fact may still lead to robustness. Details are still under investigation.

\section{Numerical Simulations}

\subsection{First guess for $g$}

We consider $g_{2}$ as in (2.2) and the system with 5 nodes and 9 arcs displayed in Figure 2. We take matrix $D$ as follows

$$
D=\left[\begin{array}{ccccc}
0 & 1 & 0 & 0 & 0 \\
0 & 0 & 0.5 & 0 & 0 \\
-0.1 & 0 & 0.5 & 0 & 0 \\
-0.2 & 0 & 0 & 0 & 0 \\
0 & 0 & 0 & 0 & 0 \\
0 & 0 & 0.5 & 0 & 0 \\
0.1 & 0 & 0 & 1 & 0 \\
0.6 & 1 & 1 & 0 & 0 \\
0.4 & 0 & 0 & 1 & 1
\end{array}\right]
$$

and $\bar{\omega}=\left[\begin{array}{llllll}0 & 1 & 1 & 1 & 1\end{array}\right]^{\prime}$.

Figure 3 displays the time plot of the state trajectory. We have 9 state variables (as many as the arcs of the network). Chattering around the hyperplane $\mathcal{H}$ is due to the discontinuous behavior of $\omega$ that jumps between $\bar{\omega}$ and $-\bar{\omega}$.

\subsection{Second, third, and fourth guesses for $g$}

In this section we show numerical examples and plot state trajectories related to the second, third and fourth guesses for $g$.

We consider $g_{3}$ as in (2.3) and again the system with 1 node and 2 arcs displayed in Figure 1 . The incidence matrix is $B=\left[\begin{array}{ll}1 & 1\end{array}\right]$ and we take matrix $D=\left[\begin{array}{ll}1 / 2 & 1 / 2\end{array}\right]^{\prime}$. 


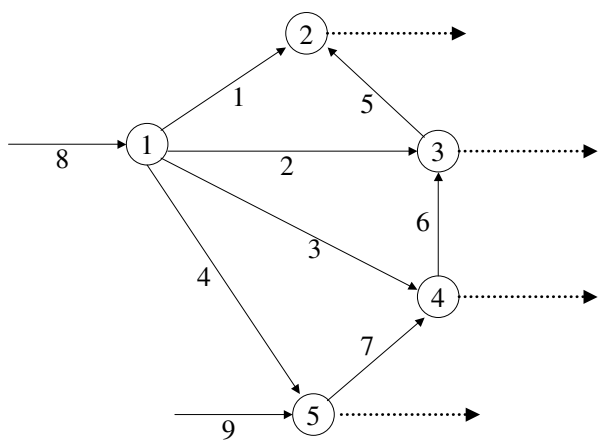

Figure 2. Example of a system with 5 nodes and 9 arcs.

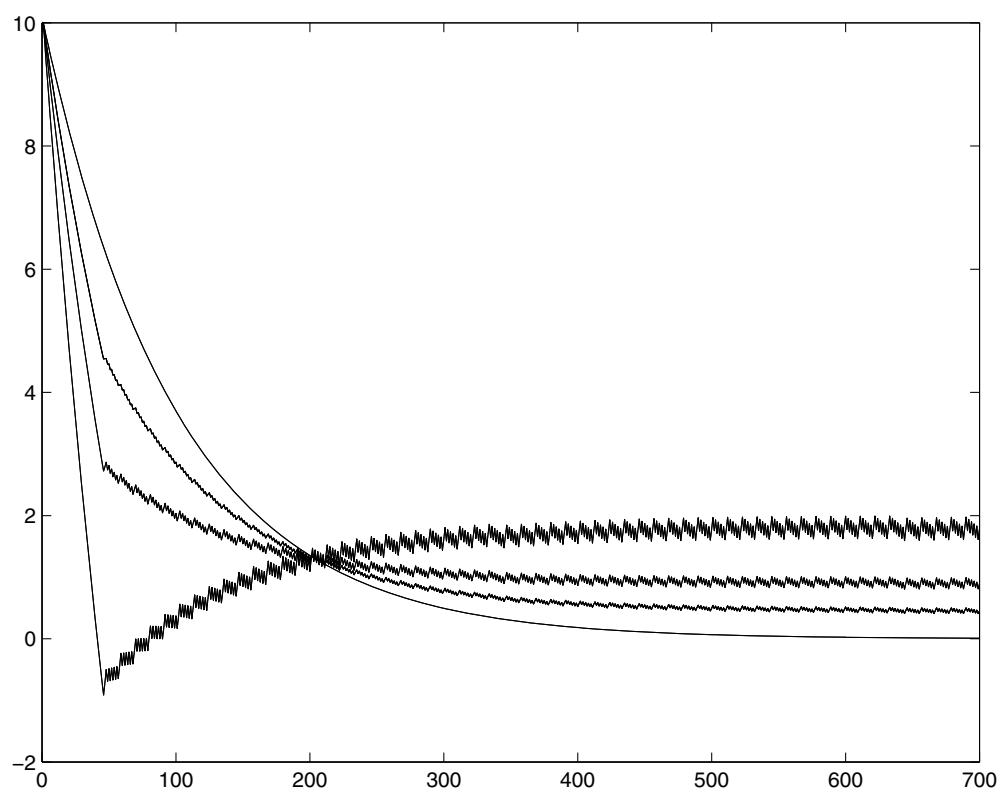

Figure 3. State trajectory for the system of Figure 2. Chattering around the hyperplane $\mathcal{H}$ is due to the discontinuous behavior of $\omega$ that jumps between $\bar{\omega}$ and $-\bar{\omega}$.

In Figures 4 and 5 (top left), we plot the state trajectory in the phase plane for the two cases $C_{2}>0$ and $C_{2}<0$ and from different initial states (circles) as

$$
z(0)=\left[\begin{array}{l}
5 \\
5
\end{array}\right],\left[\begin{array}{c}
5 \\
-5
\end{array}\right],\left[\begin{array}{l}
-5 \\
-5
\end{array}\right],\left[\begin{array}{l}
-5 \\
-5
\end{array}\right],\left[\begin{array}{l}
7.5 \\
2.5
\end{array}\right],\left[\begin{array}{c}
2.5 \\
-5
\end{array}\right],\left[\begin{array}{c}
-2.5 \\
-5
\end{array}\right],\left[\begin{array}{l}
-5 \\
2.5
\end{array}\right],\left[\begin{array}{l}
2.5 \\
7.5
\end{array}\right] .
$$

In evidence the two equilibria $\pm \frac{D \bar{\omega}}{k}= \pm\left[\begin{array}{ll}10 & 10\end{array}\right]^{\prime}$ (star).

When $C_{2}<0$ (see Fig. 4) we have $\omega=-\bar{\omega}$ and the trajectories remain in the same half-space reaching the nearest equilibrium (lying on the same half-space). No switching behavior of $\omega$ occurs as the state do not cross the hyperplane $\zeta \cdot D \bar{\omega}=0$.

Differently, when $C_{2}>0$ (see Fig. 5 (top left)) we have $\omega=\bar{\omega}$ and the trajectories try to leave one half-space to reach the equilibrium of the other half-space. The trajectory do not reach the equilibrium because of the switching behavior of $\omega$ across the hyperplane $\zeta \cdot D \bar{\omega}=0$. 


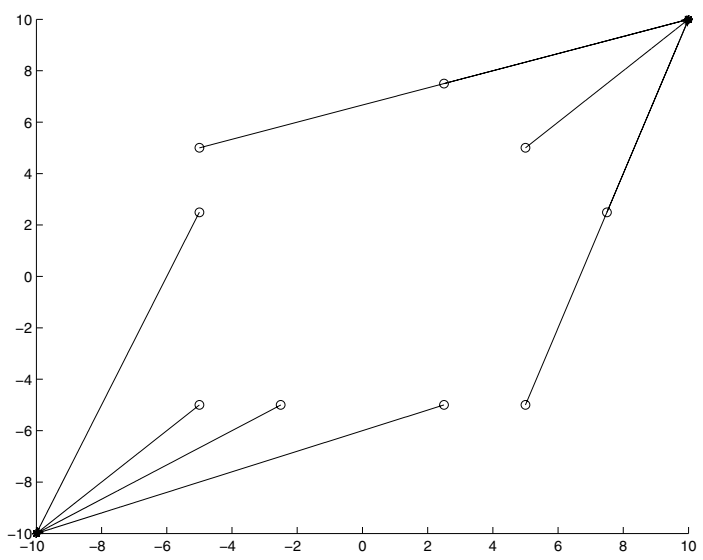

FIgURE 4. State trajectory in the phase plane from different initial states (circles) for $g_{3}$ as in (2.3) and $C_{2}<0$. In evidence the two equilibria $\pm \frac{D \bar{\omega}}{k}= \pm\left[\begin{array}{ll}10 & 10\end{array}\right]^{\prime}$ (star).
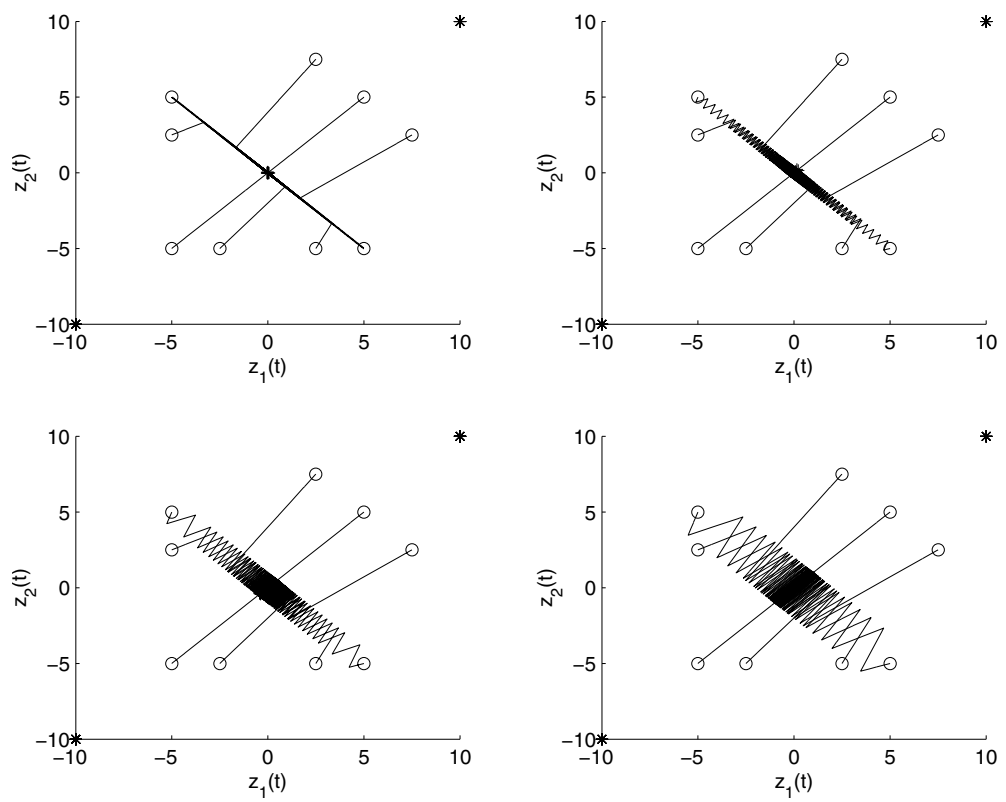

FiguRE 5. State trajectory in the phase plane from different initial states (circles). In evidence the two equilibria $\pm \frac{D \bar{\omega}}{k}= \pm\left[\begin{array}{ll}10 & 10\end{array}\right]^{\prime}$ (star). (Top left) $g_{3}$ as in (2.3) and $C_{2}>0$; (top right) $g_{4}$ as in (2.4) and $\epsilon=0.2$; (bottom left) $g_{4}$ as in (2.4) and $\epsilon=0.5$; (bottom right) $g_{4}$ as in (2.4) and $\epsilon=1$.

Figure 5 also displays the state trajectories when $g_{4}$ is as in (2.4) and demand performs according to the delayed thermostat behavior. We considered different values of $\epsilon$ : (top right) $\epsilon=0.2$; (bottom left) $\epsilon=0.5$; (bottom right) $\epsilon=1$.

Note that trajectories for the fourth guess can be omitted as, apart from the switching variable $\sigma$, they are exactly the same as the ones obtained for the third guess.

Finally, we carried out simulations for the system with 5 nodes and 9 arcs displayed in Figure 2 when $g_{4}$ is as in (2.4) and demand performs according to the delayed thermostat behavior. Again, we have 9 state variables (as many as the arcs of the network). Figure 6 displays the time plot of the state trajectory for different values 

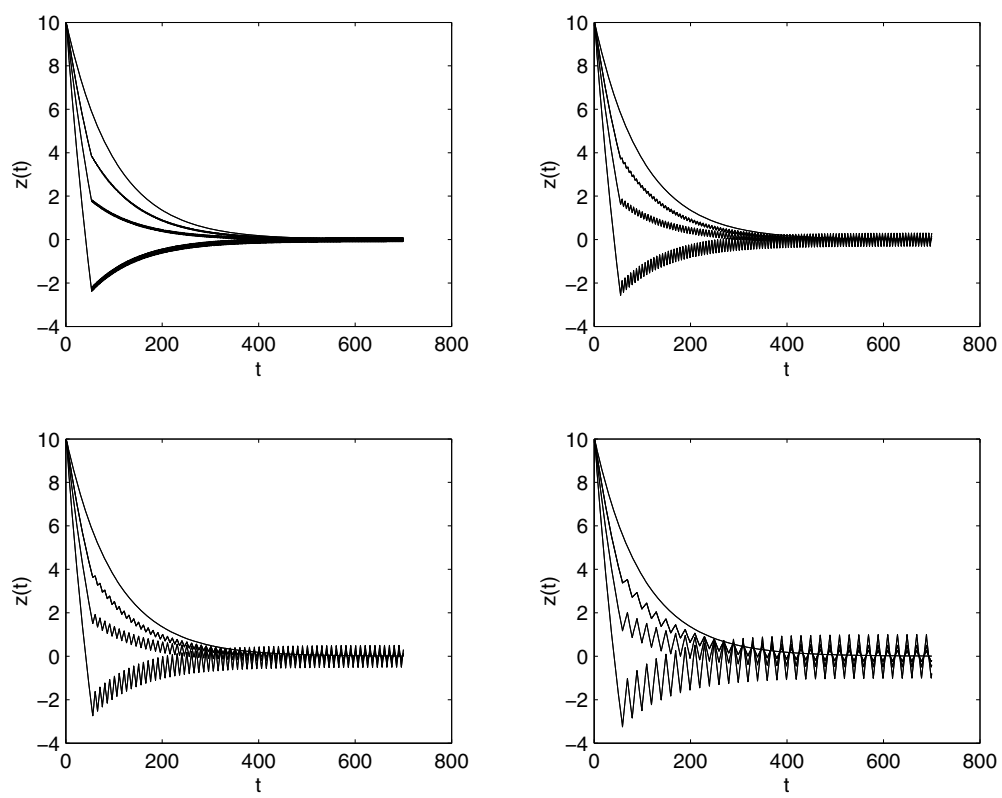

Figure 6. State trajectory for the system of Figure 2 for different values of $\epsilon$ : (top left) $\epsilon=0$ and $g_{3}$ as in $(2.3)$; (top right) $\epsilon=0.8$; (bottom left) $\epsilon=2$; (bottom right) $\epsilon=5$. Chattering around the hyperplane $\mathcal{H}$ increases with $\epsilon$.

of $\epsilon$ : (top left) $\epsilon=0$ and $g_{3}$ as in (2.3), (top right) $\epsilon=0.8$; (bottom left) $\epsilon=2$; (bottom right) $\epsilon=5$. Chattering around the hyperplane $\mathcal{H}$ is due to the discontinuous behavior of $\omega$ that jumps between $\bar{\omega}$ and $-\bar{\omega}$. Amplitude of chattering increases with $\epsilon$. Again, the above trajectories are unchanged if we choose $g_{5}$ as in (2.5).

\section{Appendix}

\subsection{Some facts on Hamilton-Jacobi, viscosity solutions and control problems}

For the general theory and results concerning viscosity solutions for Hamilton-Jacobi equations see Bardi and Capuzzo Dolcetta [4]. Here, we state and prove some relatively new results exposed in a manner which is suitable for our purposes.

\subsubsection{Viscosity solutions with boundary conditions in the viscosity sense}

Let us give

$\Omega \subset \mathbb{R}^{n}$ open, bounded, satisfying a uniform cone property (see [4] eq: IV (5.21)),

$F: \mathbb{R}^{n} \times \mathbb{R}^{n} \rightarrow \mathbb{R}$ a continuous function, $\quad \xi: \partial \Omega \rightarrow \mathbb{R}$ a function.

We say that a continuous function $v: \bar{\Omega} \rightarrow \mathbb{R}$ is a viscosity solution of the boundary value problem, with boundary condition in the viscosity sense,

$$
\begin{cases}v(x)+F(x, \nabla v(x))=0 & \text { in } \Omega \\ v=\xi & \text { on } \partial \Omega\end{cases}
$$


if $v$ is a viscosity solution of the Hamilton-Jacobi equation in $\Omega$ (i.e. the first line) and, for every $x \in \partial \Omega$ and for every continuously differentiable function $\varphi: \bar{\Omega} \rightarrow \mathbb{R}$ we have

$$
\begin{aligned}
& v-\varphi \text { has a local maximum in } x \text { with respect to } \bar{\Omega} \Longrightarrow \min \left(v(x)-\xi_{*}(x), v(x)+F(x, \nabla \varphi(x))\right) \leq 0 ; \\
& v-\varphi \text { has a local minimum in } x \text { with respect to } \bar{\Omega} \Longrightarrow \max \left(v(x)-\xi^{*}(x), v(x)+F(x, \nabla \varphi(x))\right) \geq 0,
\end{aligned}
$$

where $\xi_{*}$ and $\xi^{*}$ are respectively the lower semicontinuous envelope and the upper semicontinuous envelope:

$$
\xi_{*}(x)=\liminf _{y \rightarrow x, y \in \partial \Omega} \xi(y), \quad \xi^{*}(x)=\limsup _{y \rightarrow x, y \in \partial \Omega} \xi(y), \quad \forall x \in \partial \Omega .
$$

\subsubsection{Optimal control problems with exit-time}

Here, to not introduce much more notation, $\mathcal{W}$ and $W$ are the same as in (0.4), (0.7). Moreover $\Omega$ and $\xi$ are as in (6.1), but we further assume that there exist $m C^{2}$-functions $\theta_{i}, i=1, \ldots, m$, such that

$$
\Omega=\left\{x \in \mathbb{R}^{n} \mid \theta_{i}(x)>0 \forall i=1, \ldots, m\right\} .
$$

Moreover, we use the following notation

$$
(\partial \Omega)_{1}=\left\{x \in \mathbb{R}^{n} \mid \theta_{1}(x)=0\right\} \subseteq \partial \Omega, \quad(\partial \Omega)_{\hat{1}}=\left\{x \in \mathbb{R}^{n} \mid \theta_{i}(x)=0 i=2, \ldots, m\right\} \subseteq \partial \Omega,
$$

and suppose that $\xi: \partial \Omega \rightarrow \mathbb{R}$ is continuous on $(\partial \Omega)_{1}$ and on $(\partial \Omega)_{\hat{1}}$ separately and it is globally upper semicontinuous on $\partial \Omega$, i.e. for all $x \in(\partial \Omega)_{\hat{1}} \cap(\partial \Omega)_{1}$

$$
\lim _{y \rightarrow x, y \in(\partial \Omega)_{\hat{1}}} \xi(y)=\xi_{*}(x) \leq \xi^{*}(x)=\lim _{y \rightarrow x, y \in(\partial \Omega)_{1}} \xi(y) .
$$

Finally, $f: \mathbb{R}^{n} \times \mathcal{W} \rightarrow \mathbb{R}^{n}$ and $\ell: \mathbb{R}^{n} \times \mathcal{W} \rightarrow \mathbb{R}$ are two bounded Lipschitz continuous functions. We now consider the controlled dynamical system in $\mathbb{R}^{n}$

$$
\left\{\begin{array}{l}
y^{\prime}(t)=f(y(t), w(t)) \quad t>0 \\
y(0)=x \in \bar{\Omega}
\end{array}\right.
$$

and the payoff (here and in the sequel, by continuity, $\xi_{*}(x)=\xi^{*}(x)=\xi(x)$ if $x \notin(\partial \Omega)_{1} \cap(\partial \Omega)_{\hat{1}}$ )

$$
J(x, w)=\int_{0}^{t_{x, w}} \mathrm{e}^{-t} \ell(y(t), w(t)) \mathrm{d} t+\mathrm{e}^{-t_{x, w}} \xi^{*}\left(y\left(t_{x, w}\right)\right),
$$

where $y(\cdot)$ is the unique trajectory of (6.5) with $w \in W$, and $t_{x, w}$ is the first exit time of the trajectory from $\bar{\Omega}$ :

$$
t_{x, w}=\inf \{t \geq 0 \mid y(t) \notin \bar{\Omega}\},
$$

with the convention $\inf \emptyset=+\infty$ (and $\left.\mathrm{e}^{-\infty} \xi=0\right)$.

Proposition 6.1. Standing all the above hypotheses, if on $(\partial \Omega)_{1}$ the dynamics is totally controllable i.e.:

$\forall x \in(\partial \Omega)_{1}, \exists \omega_{1}, \omega_{2} \in \mathcal{W}$ such that: $f\left(x, \omega_{1}\right)$ is strictly entering in $\Omega$ at $x$ and $f\left(x, \omega_{2}\right)$ is strictly entering in the complementary of $\bar{\Omega}$ at $x$,

on $(\partial \Omega)_{\hat{1}}$ we have only strictly inward admissible fields, i.e.:

$$
f(x, \omega) \text { is strictly entering in } \Omega \text { at } x \forall x \in(\partial \Omega)_{\hat{1}}, \forall \omega \in \mathcal{W},
$$


and moreover if for every $x \in(\partial \Omega)_{\hat{1}}$ there exists a control $w \in W$ such that the corresponding trajectory starting from $x$ reaches $(\partial \Omega)_{1}$ in a lap of time $t$ satisfying

$$
t \leq C \operatorname{dist}\left(x,(\partial \Omega)_{1}\right),
$$

with $C>0$ independent on $x$, then the value function of the maximization problem

$$
v(x)=\sup _{w \in W} J(x, w)
$$

is continuous in $\bar{\Omega}$, and it is the unique viscosity solution of the Hamilton-Jacobi-Bellman boundary value problem (with boundary condition in the viscosity sense)

$$
\begin{cases}v(x)+\min _{\omega \in \mathcal{W}}\{-f(x, \omega) \cdot \nabla v(x)-\ell(x, \omega)\}=0 & \text { in } \Omega \\ v=\xi^{*} & \text { on } \partial \Omega,\end{cases}
$$

satisfying the condition

$$
v(x) \geq \xi^{*}(x) \quad \forall x \in(\partial \Omega)_{1} \cap(\partial \Omega)_{\hat{1}} .
$$

Proof. The proof suitably adapts standard techniques to our situation. We do not report it. However, we are going to report a sketched proof for the analogous case of differential games.

\subsubsection{Differential games with exit-time}

Here, again, $\mathcal{W}, W$ are as in (0.4), (0.7). Moreover $\mathcal{U}$ and $U$ are as in (0.3), (0.7), $\Gamma$ is as in (0.8), $\Omega$ and $\xi$ are as in (6.1), satisfying also (6.2), (6.3), and moreover $\xi: \partial \Omega \rightarrow \mathbb{R}$ is continuous on $(\partial \Omega)_{1}$ and on $(\partial \Omega)_{\hat{1}}$ separately and it is globally lower semicontinuous on $\partial \Omega$, i.e. for all $x \in(\partial \Omega)_{\hat{1}} \cap(\partial \Omega)_{1}$

$$
\lim _{y \rightarrow x, y \in(\partial \Omega)_{1}} \xi(y)=\xi_{*}(x) \leq \xi^{*}(x)=\lim _{y \rightarrow x, y \in(\partial \Omega)_{\hat{1}}} \xi(y) .
$$

Note the difference between (6.12) and (6.4): the role of $(\partial \Omega)_{1}$ and $(\partial \Omega)_{\hat{1}}$ are mutually exchanged. Finally, $\tilde{f}: \mathbb{R}^{n} \times \mathcal{U} \times \mathcal{W} \rightarrow \mathbb{R}^{n}$ and $\tilde{\ell}: \mathbb{R}^{n} \times \mathcal{U} \times \mathcal{W} \rightarrow \mathbb{R}$ are two Lipschitz continuous functions. We now consider the controlled dynamical system in $\mathbb{R}^{n}$ and the payoff

$$
\begin{gathered}
\left\{\begin{array}{l}
y^{\prime}(t)=\tilde{f}(y(t), \gamma[w](t), w(t)) \quad t>0, \\
y(0)=x \in \bar{\Omega},
\end{array}\right. \\
\tilde{J}_{*}(x, \gamma, w)=\int_{0}^{t_{x, \gamma, w}} \mathrm{e}^{-t} \tilde{\ell}(y(t), \gamma[w](t), w(t)) \mathrm{d} t+\mathrm{e}^{-t_{x, \gamma, w}} \xi_{*}\left(y\left(t_{x, \gamma, w}\right)\right),
\end{gathered}
$$

where $y(\cdot)$ is the unique trajectory of (6.13) with $\gamma \in \Gamma w \in W$, and $t_{x, \gamma, w}$ is the first exit time of the trajectory from $\bar{\Omega}$ (similarly defined as in (6.6)).

Proposition 6.2. Standing the above hypotheses, if the dynamics is totally controllable by the first player (the minimizing one) on the points of the boundary i.e.

$\forall x \in \partial \Omega, \exists \mu_{1}, \mu_{2} \in \mathcal{U}$ such that, $\forall \omega \in \mathcal{W}: \tilde{f}\left(x, \mu_{1}, \omega\right)$ is strictly entering in $\Omega$ at $x$ and

$\tilde{f}\left(x, \mu_{2}, \omega\right)$ is strictly entering in the complementary of $\bar{\Omega}$ at $x$,

moreover if for every $x \in(\partial \Omega)_{\hat{1}}$ there exists a strategy $\bar{\gamma} \in \Gamma$ such that, for every $w \in W$, the corresponding trajectory starting from $x$ reaches $(\partial \Omega)_{1}$ in a lap of time $t$ satisfying

$$
t \leq C \operatorname{dist}\left(x,(\partial \Omega)_{1}\right)
$$


with $C>0$ independent on $w \in W$, then the (lower) value function of the minmax problem

$$
\tilde{v}(x)=\inf _{\gamma \in \Gamma} \sup _{w \in W} \tilde{J}_{*}(x, \gamma, w)
$$

is continuous in $\bar{\Omega}$, and it is the unique continuous viscosity solution of the Hamilton-Jacobi-Isaacs boundary value problem (with boundary condition in the viscosity sense)

$$
\begin{cases}\tilde{v}(x)+\min _{\omega \in \mathcal{W}} \max _{\mu \in \mathcal{U}}\{-\tilde{f}(x, \mu, \omega) \cdot \nabla \tilde{v}(x)-\tilde{\ell}(x, \mu, \omega)\}=0 & \text { in } \Omega \\ \tilde{v}=\xi_{*} & \text { on } \partial \Omega\end{cases}
$$

satisfying the condition

$$
\tilde{v}(x) \leq \xi_{*}(x) \quad \forall x \in(\partial \Omega)_{1} \cap(\partial \Omega)_{\hat{1}} .
$$

Proof. $\tilde{v}$ is continuous. The continuity of $\tilde{v}$ comes from (6.15), (6.16), from the fact that $\xi$ is separately continuous on $(\partial \Omega)_{1}$ and $(\partial \Omega)_{\hat{1}}$, and also from the fact that $\xi_{*}$ is continuous on $(\partial \Omega)_{1} \cap(\partial \Omega)_{\hat{1}}($ see $(6.12))$. In particular note that (6.16) guarantees the (controlled in time) reachability of $(\partial \Omega)_{1}$, where the values of the exit cost $\xi$ are (probably) lower than the values on $(\partial \Omega)_{\hat{1}}$, at least for points near to $(\partial \Omega)_{1} \cap(\partial \Omega)_{\hat{1}}$. Indeed, adapting a result due to Soner [24] (see also Bagagiolo and Bardi [2] for a generalization to a polytopic case as (6.2)), there exist a time $\tau>0$ and a constant $\alpha>0$ (both depending only on $\Omega$ and on $\tilde{f}$ via (6.15)) such that, for every $x \in \bar{\Omega}$ and for every $u \in U$, there exists a control $\bar{u} \in U$ such that

$$
\begin{aligned}
& y_{x}(t ; \bar{u}, w) \in \bar{\Omega}, \quad \forall 0 \leq t \leq \tau, \forall w \in W \\
& \left|J_{\tau}(x, \bar{u}, w)-J_{\tau}(x, u, w)\right| \leq \alpha \sup _{0 \leq t \leq \tau} \operatorname{dist}\left(y_{x}(t ; u, w), \bar{\Omega}\right), \quad \forall w \in W
\end{aligned}
$$

where $y_{x}(\cdot ; \bar{u}, w)$ and $y_{x}(\cdot ; u, w)$ are respectively the trajectories of $(6.13)$ with $\bar{u}$ and $u$ as first control $(i . e . \gamma[w] \equiv$ $\bar{u}, \gamma[w] \equiv u$, respectively), and, in general, for $t \geq 0, J_{t}$ is the corresponding cost given only by the integral part of (6.14) up to the time $t$, independently whether the trajectory stays inside $\bar{\Omega}$ or not. From (6.19), and standard estimates on the trajectories, we get the following

$$
\begin{aligned}
& \forall T>0, \exists C_{T}>0 \text { such that } \forall x, y, \in \bar{\Omega}, \forall(u, w) \in U \times W \\
& \text { with } y_{y}(t ; u, w) \in \bar{\Omega} \forall 0 \leq t \leq T, \quad \exists \bar{u} \in U \text { such that } \\
& \left\|y_{x}(t ; \bar{u}, w)-y_{x}(t ; u, w)\right\| \leq C_{T}\|x-y\| \forall t \in[0, T] \\
& \left\|y_{x}(t ; \bar{u}, w)-y_{y}(t ; u, w)\right\| \leq C_{T}\|x-y\| \forall t \in[0, T] \\
& \left|J_{T}(x, \bar{u}, w)-J_{T}(y, u, w)\right| \leq C_{T}\|x-y\| .
\end{aligned}
$$

Now, from (6.20), from the reachability condition (6.16), and again from the controllability condition (6.15), and from standard inequalities on the trajectories, we then get the following

$$
\begin{aligned}
& \forall \delta>0 \exists C_{\delta}>0 \text { such that } \forall x, y \in \bar{\Omega}, \forall \gamma \in \Gamma, \exists \bar{\gamma} \in \Gamma \text { such that } \forall w \in W \\
& \left|J_{*}(x, \bar{\gamma}, w)-J_{*}(y, \gamma, w)\right| \leq C_{\delta}\|x-y\|+\delta .
\end{aligned}
$$

To obtain (6.21), observe that all the estimates in (6.20) are independent on $w \in W$, and take $T>0$ such that the possible remaining part of the cost $J_{*}$ in the time interval $(T,+\infty)$ is certainly less than $\delta / 2$ for every initial point $x$ and couple of controls $(u, w)$. Then construct the strategy $\bar{\gamma}$ defining $\bar{\gamma}[w]$ by observing the trajectory $y_{y}(\cdot ; \gamma[w], w)$ and making $y_{x}(\cdot ; \bar{\gamma}[w], w)$ have suitable behavior and cost: use (6.15) to exit from $\bar{\Omega}$ if necessary; use (6.20) to remain inside $\bar{\Omega}$ if necessary; use (6.16) to reach $(\partial \Omega)_{1}$ as final exit point if necessary. From (6.21), 


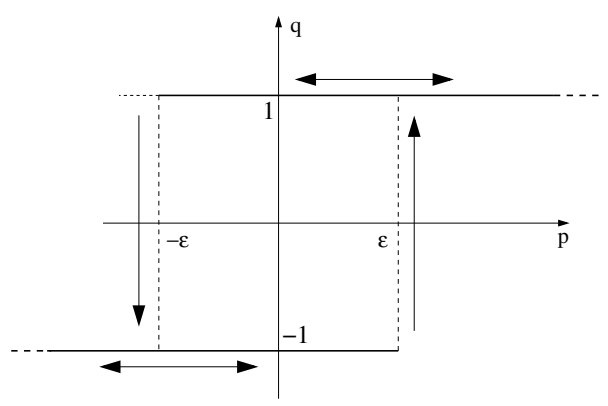

Figure 7. Delayed thermostat with thresholds $\pm \varepsilon$.

we easily get the continuity of $\tilde{v}$. It is sufficient to argue by absurd and suppose that there exist a point $x \in \bar{\Omega}$, a sequence $\left\{y_{n}\right\}_{n} \subset \bar{\Omega}$ converging to $x$, and $\varepsilon>0$ such that $\left|\tilde{v}(x)-\tilde{v}\left(y_{n}\right)\right| \geq \varepsilon$ for all $n \in \mathbb{N}$.

$\tilde{v}$ solves (6.17), (6.18). This fact is checked by standard techniques. In particular, observe that by the controllability hypothesis (6.15), $\tilde{v}$ certainly satisfies (6.18).

$\tilde{v}$ is the unique solution of (6.17). We argue as in Bardi and Capuzzo Dolcetta [4] (Chap. V, Thm. 4.17). The only difference here is that the boundary datum is discontinuous. However, the only possible discontinuous points are in $(\partial \Omega)_{1} \cap(\partial \Omega)_{\hat{1}}$. But there, condition (6.18) guarantees the applicability of the argument.

Remark 6.3. Differential games with exit time and/or state-constraints present, in general, the problem of devolving the responsibility of exit and/or respecting the state-constraints to both players (see Evans and Ishii [17], Koike [19], Bardi et al. [5], Cardialaguet et al. [11]). In the present case, the controllability condition (6.15) and the fact that we are only concerned with the lower value (first minimizing, then maximizing), make all the responsibility and the faculty of exit and/or respecting the state-constraint be up to the first player only (the minimizing one). Uniqueness results for first order Hamilton-Jacobi equations with discontinuous boundary datum and/or discontinuous Hamiltonians are in general hard to get, see for instance Soravia [23] and Garavello and Soravia [18]. In the present paper, conditions (6.11), (6.18), and the approximation of the discontinuity by the delayed relay are of course useful. However, our goal is not to give a general result of uniqueness, but instead to have suitable uniqueness results in order to recognize given functions as the values of the optimal control and of the differential game. This is what we just do in Section 4.

\subsection{On the delayed thermostat}

For more details on this subject we refer to Visintin [25]. Let us consider a continuous input $p:[0,+\infty[\rightarrow \mathbb{R}$, a discontinuous output $q:[0,+\infty[\rightarrow\{-1,1\}$, and two different thresholds for the values of $p$, let us say $-\varepsilon$ and $\varepsilon$, with $\varepsilon>0$, for which $q$ respectively switches "down" from +1 to -1 , and "up" from -1 to +1 . We define

$$
\overline{\mathcal{O}}:=(]-\infty,+\varepsilon] \times\{-1\}) \cup\left(\left[-\varepsilon,+\infty[\times\{1\}) \subset \mathbb{R}^{2}=: \overline{\mathcal{O}}_{-1} \cup \overline{\mathcal{O}}_{1},\right.\right.
$$

and we can think to the delayed switching as the evolution of the couple $(p(\cdot), q(\cdot))$ on the set $\overline{\mathcal{O}}$ with a suitable switching rule for switching from one branch to the other (see Fig. 7). More in details, we say that $q$ is the output of the delayed switching rule (or "delayed thermostat", or "delayed relay") with thresholds $-\varepsilon, \varepsilon$, input $u$, and initial state $q_{0} \in\{-1,1\}$, and we write $q(t)=h_{\varepsilon}\left[p, q_{0}\right](t) \forall t \geq 0$, if (here $\delta$ is any positive number)

(i) $(p(t), q(t)) \in \overline{\mathcal{O}} \quad \forall t \geq 0, \quad q(0)=q_{0},\left(p(0), q_{0}\right) \in \overline{\mathcal{O}}$

(ii) $q(t)=1, p(\cdot) \geq-\varepsilon$ in $[t, t+\delta] \Longrightarrow q(\cdot)$ is constant in $[t, t+\delta]$,

(iii) $q(t)=-1, p(\cdot) \leq \varepsilon$ in $[t, t+\delta] \Longrightarrow q(\cdot)$ is constant in $[t, t+\delta]$.

Such conditions say that $q$ switches if and only if the couple $(p, q)$ is on one of the two switching points $(-\varepsilon, 1)$ and $(\varepsilon,-1)$, and the input $p$ crosses the threshold $\pm \varepsilon$ (decreasing if the threshold is $-\varepsilon$, increasing if it is $\varepsilon$ ). 
According to this rule, a switching instant $t \geq 0$ is a time such that $q(t)=\bar{q} \in\{1,-1\}$, and $q \equiv-\bar{q}$ immediately after $t$ (for instance in $] t, t+\delta]$ ). Referring to exit-time problems, we can say that, if at a time $t$ we have, for instance, $(p(t), q(t))=(p(t), 1)$ then certainly $p(t) \geq-\varepsilon$ and a possible subsequent switching time $\tau \in[t,+\infty[$ is exactly the first exit time of the trajectory $p(\cdot)$ from the closed set $[-\varepsilon,+\infty[$, which is similarly defined as in (6.6). In particular, for the same example, the fact that $p(\bar{t})=-\varepsilon$ for some $\bar{t} \geq t$, does not imply any switching if the threshold is not crossed (i.e. if $p \nless-\varepsilon$ immediately after $\bar{t}$ ). After any switching instant $t$, $q$ cannot immediately switch back, because $p$ has to reach the other threshold. This implies the existence of exactly one output, even for fast oscillating inputs, i.e. no Zeno phenomenon.

\section{REFERENCES}

[1] F. Bagagiolo, Minimum time for a hybrid system with thermostatic switchings, in Hybrid Systems: Computation and Control, A. Bemporad, A. Bicchi and G. Buttazzo Eds., Lect. Notes Comput. Sci. 4416, Springer-Verlag, Berlin, Germany (2007) $32-45$.

[2] F. Bagagiolo and M. Bardi, Singular perturbation of a finite horizon problem with state-space constraints. SIAM J. Contr. Opt. 36 (1998) 2040-2060.

[3] F. Bagagiolo and D. Bauso, Robust optimality of linear saturated control in uncertain linear network flows, in Decision and Control, 2008, CDC 2008, 47th IEEE Conference (2008) 3676-3681.

[4] M. Bardi and I. Capuzzo Dolcetta, Optimal Control and Viscosity Solutions of Hamilton-Jacobi-Bellman Equations. Birkhäuser, Boston, USA (1997).

[5] M. Bardi, S. Koike and P. Soravia, Pursuit-evasion games with state constraints: dynamic programming and discrete-time approximation. Discrete Contin. Dyn. Syst. 6 (2000) 361-380.

[6] D. Bauso, F. Blanchini and R. Pesenti, Robust control policies for multi-inventory systems with average flow constraints. Automatica 42 (2006) 1255-1266.

[7] A. Bemporad, M. Morari, V. Dua and E.N. Pistikopoulos, The explicit linear quadratic regulator for constrained systems. Automatica 38 (2002) 320.

[8] A. Ben Tal and A. Nemirovsky, Robust solutions of uncertain linear programs. Oper. Res. 25 (1998) 1-13.

[9] D.P. Bertsekas and I. Rhodes, Recursive state estimation for a set-membership description of uncertainty. IEEE Trans. Automatic Control 16 (1971) 117-128.

[10] D. Bertsimas and A. Thiele, A robust optimization approach to inventory theory. Oper. Res. 54 (2006) 150-168.

[11] P. Cardialaguet, M. Quincampoix and P. Saint-Pierre, Pursuit differential games with state constraints. SIAM J. Contr. Opt. 39 (2001) 1615-1632.

[12] J. Casti, On the general inverse problem of optimal control theory. J. Optim. Theory Appl. 32 (1980) 491-497.

[13] X. Chen, M. Sim, P. Sun and J. Zhang, A linear-decision based approximation approach to stochastic programming. Oper. Res. 56 (2008) 344-357.

[14] M.G. Crandall, L.C. Evans and P.L. Lions, Some properties of viscosity solutions of Hamilton-Jacobi equations. Trans. Amer. Math. Soc. 282 (1984) 487-502.

[15] S. Dharmatti and M. Ramaswamy, Zero-sum differential games involving hybrid controls. J. Optim. Theory Appl. 128 (2006) $75-102$.

[16] R.J. Elliot and N.J. Kalton, The existence of value in differential games, Mem. Amer. Math. Soc. 126. AMS, Providence, USA (1972).

[17] L.C. Evans and H. Ishii, Differential games and nonlinear first order PDE on bounded domains. Manuscripta Math. 49 (1984) 109-139.

[18] M. Garavello and P. Soravia, Representation formulas for solutions of HJI equations with discontinuous coefficients and existence of value in differential games. J. Optim. Theory Appl. 130 (2006) 209-229.

[19] S. Koike, On the state constraint problem for differential games. Indiana Univ. Math. J. 44 (1995) 467-487.

[20] O. Kostyukova and E. Kostina, Robust optimal feedback for terminal linear-quadratic control problems under disturbances. Math. Program. 107 (2006) 131-153.

[21] V.B. Larin, About the inverse problem of optimal control. Appl. Comput. Math 2 (2003) 90-97.

[22] T.T. Lee and G.T. Liaw, The inverse problem of linear optimal control for constant disturbance. Int. J. Control 43 (1986) 233-246.

[23] P. Soravia, Boundary value problems for Hamilton-Jacobi equations with discontinuous Lagrangian. Indiana Univ. Math. J. 51 (2002) 451-477.

[24] H.M. Soner, Optimal control problems with state-space constraints I. SIAM J. Contr. Opt. 31 (1986) 132-146.

[25] A. Visintin, Differential Models of Hysteresis. Springer-Verlag, Berlin, Germany (1996). 